\title{
A Review of Basal-Bolus Therapy Using Insulin Glargine and Insulin Lispro in the Management of Diabetes Mellitus
}

\author{
Riccardo Candido $\cdot$ Kathleen Wyne $\cdot$ Ester Romoli
}

\begin{abstract}
Basal-bolus therapy (BBT) refers to the combination of a long-acting basal insulin with a rapid-acting insulin at mealtimes. Basal insulin glargine $100 \mathrm{U} / \mathrm{mL}$ and prandial insulin lispro have been available for many years and there is a substantial evidence base to support the efficacy and safety of these agents when they are used in BBT or basal-plus therapy for patients with type 1 or type 2 diabetes mellitus (T1DM, T2DM). With the growing availability of alternative insulins for use in such regimens, it seems timely to review the data regarding BBT with insulin glargine $100 \mathrm{U} / \mathrm{mL}$ and insulin lispro. In patients with T1DM, BBT with insulin glargine plus insulin lispro provides similar or better glycemic control and leads to less nocturnal hypoglycemia compared to BBT using human insulin as the basal and/or prandial
\end{abstract}

Enhanced digital features To view enhanced digital features for this article go to https://doi.org/10.6084/ m9.figshare.6040796.

R. Candido ( $\square)$

Diabetes Centre District 3, Azienda Sanitaria

Universitaria Integrata di Trieste, Via Puccini 48/50,

34100 Trieste, Italy

e-mail: riccardocandido@yahoo.it

K. Wyne

The Ohio State University Wexner Medical Center, Columbus, OH 43210, USA

E. Romoli

Eli Lilly Italia SPA, via A. Gramsci 731/733, 50019

Sesto Fiorentino, Italy component, and generally provides similar glycemic control and rates of severe hypoglycemia to those achieved with insulin lispro administered by continuous subcutaneous insulin infusion (CSII). Studies evaluating BBT with insulin glargine plus insulin lispro in patients with T2DM also demonstrate the efficacy and safety of these insulins. Available data suggest that BBT with insulin glargine and insulin lispro provides similar levels of efficacy and safety in pediatric and adult populations with T1DM and in adult patients and those aged more than 65 years with T2DM. These insulin preparations also appear to be safe and effective for controlling T2DM in people of different ethnicities and in patients with T1DM or T2DM and comorbidities.

Funding: Eli Lilly and Company.

Keywords: Basal-bolus therapy; Insulin glargine; Insulin lispro; Lilly insulin glargine; Type 1 diabetes mellitus; Type 2 diabetes mellitus

\section{INTRODUCTION}

Insulin treatment of type 1 (T1DM) and type 2 (T2DM) diabetes mellitus has progressed considerably since the discovery of insulin in 1922 [1] and its subsequent purification and crystallization [2]. Initially, only animal insulin was available to diabetics, and animal insulin is 
associated with adverse effects such as insulin allergy, insulin resistance, and insulin lipodystrophy [3-5]. However, in the 1980s, recombinant DNA technology enabled the development of synthetic human insulin, which replaced animal insulin as it was found to be less likely to produce adverse effects and it could be massproduced. In the late 1980s and early 1990s, it became apparent that better glucose control would require new insulin preparations with a faster onset and shorter duration of action, enabling prandial insulin to be administered closer to mealtimes, as well as long-acting preparations with a flatter time-action profile and less variable bioavailability, including formulations suitable for once-daily dosing [6-9]. Molecular genetic techniques provided opportunities to create insulin analogues by changing the structure of the native protein and improving its therapeutic properties [8-10].

The crucial importance of an exogenous basal-bolus insulin supply to control blood glucose concentrations in patients with absolute insulin deficiency disease (i.e., T1DM) is well recognized. However, in T2DM, the initial relative insulin deficiency progresses with the decline in $\beta$-cell function, which again makes a combination of basal and prandial insulin the most effective insulin strategy $[5,11,12]$.

This article reviews the current evidence concerning a widely used basal-bolus strategy combining insulin glargine $100 \mathrm{U} / \mathrm{mL}$ with insulin lispro in patients with T1DM and T2DM. The review does not consider all insulins and, as such, does not include other established or new insulins used in basal-bolus regimens unless they have been directly compared with insulin glargine $100 \mathrm{U} / \mathrm{mL}$ plus insulin lispro. An introductory summary of key information about the individual agents leads into a discussion of their combined use in basal-bolus therapy (BBT). In addition, the use of this BBT in special populations is considered. The information provided is based on previously conducted and published studies and does not contain any studies with human participants or animals performed by any of the authors.

\section{INSULIN LISPRO}

\section{Structure, Pharmacokinetics, and Pharmacodynamics}

The first genetically engineered rapid-acting insulin analogue (RAIA) to become available was insulin lispro, which was approved for clinical use in Europe and the USA in 1996 [13]. The B26-30 region of the insulin molecule is not critical for binding to the insulin receptor, but it is important in mediating the formation of insulin dimers $[9,14]$. The insulin lispro molecule is created by reversing the normal sequence of proline at position 28 of the $B$ chain and lysine at position 29 (Fig. 1) [8]. This reversal causes a decreased tendency for selfassociation [13, 15, 16]; consequently, insulin lispro has faster absorption, higher peak serum levels, and a shorter duration of action compared with regular human insulin (RHI) $[9,13,17,18]$. In patients with T1DM treated with multiple daily injections, insulin lispro can be associated with fewer hypoglycemic events than RHI [13]. Importantly, structural modifications at these positions do not affect the receptor-binding domain of the molecule [16] or the affinity for the insulin receptor [9]. Although the affinity of insulin lispro for the insulin-like growth factor-1 (IGF-1) receptor is slightly higher than that of RHI, this difference in affinity is not large enough to cause a difference in cell-growth-stimulating activity [16]. Insulin lispro has essentially the same effect on lipogenesis as RHI [19].

Pharmacokinetic (PK)/pharmacodynamic (PD) studies indicate that the action of insulin lispro starts within $15 \mathrm{~min}$, peaks in approximately 40-90 min, and disappears within 2-4 h of subcutaneous (SC) injection $[18,20,21]$.

After insulin lispro, two other RAIAs, insulin aspart and insulin glulisine, were developed. Although studies have shown some differences in various PK parameters between RAIAs [22-26], overall there do not appear to be substantial differences in their effectiveness at controlling postprandial glucose levels and blood glucose profiles [19, 23-28]. However, there are some differences in the indications 


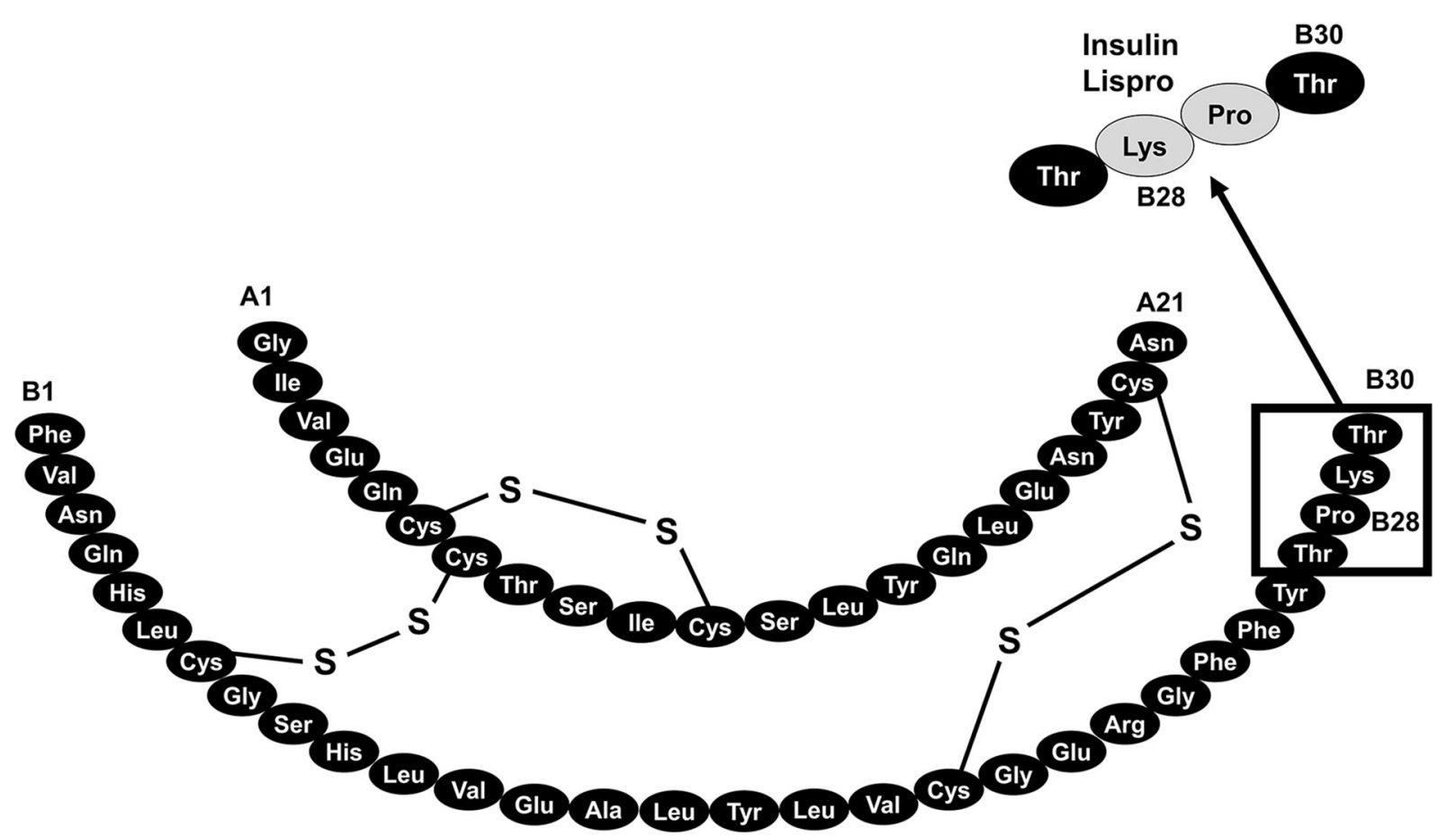

Fig. 1 Structure of insulin lispro. Ala alanine, Arg arginine, Asn asparagine, Cys cysteine, Gln glutamine, Glu glutamic acid, Gly glycine, His histidine, Ile isoleucine,

and the patient populations studied with the three analogues [29-31].

\section{Efficacy and Safety}

Insulin lispro is one of the most studied and widely used rapid-acting insulins. As expected for a RAIA, insulin lispro significantly improves postprandial blood glucose levels compared with RHI when administered as prandial insulin in conjunction with basal insulin, leading to a lower rate of hypoglycemic events [32-35]. This was observed even when insulin lispro was administered immediately before meals and RHI was injected 30-45 min before meals. However, in most cases, the beneficial effects of insulin lispro on postprandial blood glucose levels and frequency of hypoglycemic events were not accompanied by improvements in glycosylated hemoglobin (HbA1c) compared with RHI [33-35]. The most likely explanation for this is the inability of the long-acting insulins that were administered with insulin lispro
Leu leucine, Lys lysine, Phe phenylalanine, Pro proline, Ser serine, $T h r$ threonine, Tyr tyrosine, $V a l$ valine

and RHI to provide true basal coverage, meaning that increased preprandial blood glucose concentrations were present in patients on insulin lispro. Supporting this theory, a clinically and statistically significant decrease in HbA1c level was seen when insulin lispro was used in conjunction with two or more daily injections (instead of one) of neutral protamine Hagedorn (NPH) insulin [36-38].

Insulin lispro is the only rapid-acting analogue which also has a $200 \mathrm{U} / \mathrm{mL}$ formulation. Insulin lispro $200 \mathrm{U} / \mathrm{mL}$ has the same $\mathrm{PK} / \mathrm{PD}$, efficacy, and safety profiles as lispro $100 \mathrm{U} / \mathrm{mL}$ $[39,40]$, but the $200 \mathrm{U} / \mathrm{mL}$ pen had a lower glide force and was preferred by patients [41, 42].

No differences have been reported between insulin lispro and RHI regarding the likelihood of allergic reactions, nonhypoglycemic adverse events, or abnormal laboratory values $[34,35,43]$. The immunogenicity of insulin lispro is similar to that of RHI [43], and antibodies against insulin lispro rarely develop and do not affect dose requirements [43]. Interestingly, there have been reports of patients with 
severe resistance to RHI due to antibody formation that was successfully overcome by switching to insulin lispro [44-47].

\section{INSULIN GLARGINE}

\section{Structure, Pharmacokinetics, and Pharmacodynamics}

Insulin glargine is a long-acting biosynthetic human insulin analogue that was first approved for use in patients with T1DM and T2DM in the USA and Europe in 2000 (Lantus ${ }^{\circledR}$ insulin glargine) [48-50].

The structure of insulin glargine was designed by substituting an asparagine residue with a glycine at position 21 of the A chain and elongating the $\mathrm{B}$ chain at the C-terminus by adding two arginine residues (Fig. 2) [50, 51]. Modification of the B chain shifts the isoelectric point of insulin glargine, while the glycine substitution stabilizes the hexamer structure, and contributes to delayed delivery from SC depots, increased bioavailability, and maintenance of stability in acidic solutions [3, 50, 52]. After SC injection, insulin glargine precipitates in SC tissues, which delays its absorption and prolongs its duration of action [51-53]. Insulin glargine must not be mixed with other insulins, as it precipitates and the PK and PD profiles are altered.

Insulin glargine generally behaves like RHI regarding insulin receptor binding, receptor autophosphorylation, phosphorylation of signaling elements, and promotion of mitogenesis in muscle cells, apart from an increased binding affinity for the IGF-1 receptor in in-vitro (but not in cell-based) models [54, 55]. The growth-promoting activity of insulin glargine in muscle cells and its maximal metabolic activity do not differ from the effects of native human insulin, but the lipogenic activity of insulin glargine is slightly less than that of RHI [56].

The PD properties of insulin glargine differ from those of RHI. Insulin glargine exerted a glucose-lowering effect for $24 \mathrm{~h}$ after a single

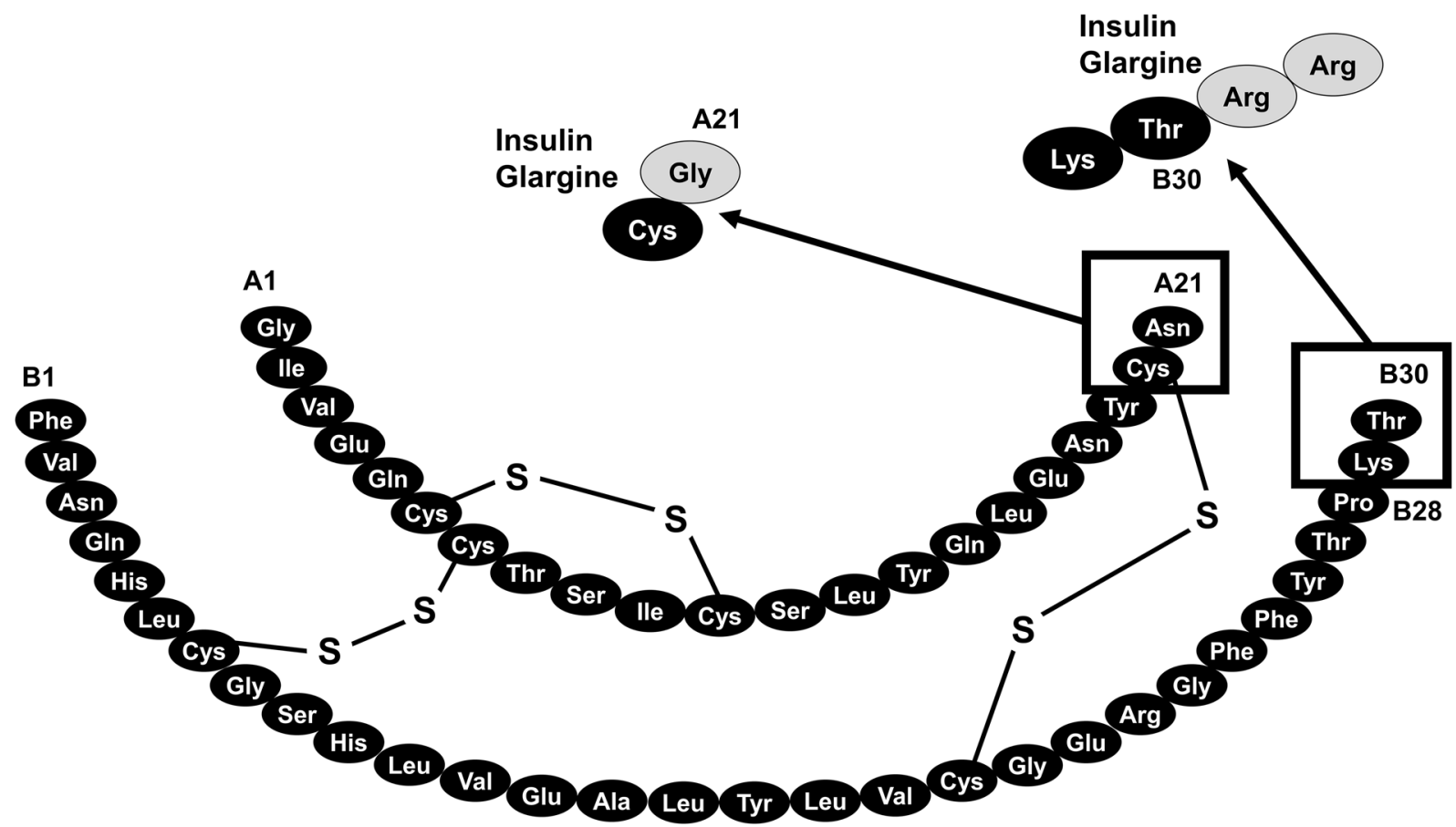

Fig. 2 Structure of insulin glargine. Ala alanine, Arg arginine, Asn asparagine, Cys cysteine, Gln glutamine, Glu glutamic acid, Gly glycine, His histidine, Ile isoleucine, Leu leucine, Lys lysine, Phe phenylalanine, Pro proline, Ser serine, $T h r$ threonine, $T y r$ tyrosine, $V$ al valine 
daily injection, without a pronounced plasma peak, and induced a smoother metabolic effect than NPH insulin $[3,57]$. Although it is recognized that the effect of NPH insulin can vary with injection site, changes in injection site do not alter the time-action profile of insulin glargine $[3,52]$.

\section{Efficacy and Safety}

One of the first studies to evaluate insulin glargine showed that once-daily injections of this analogue in patients with T1DM who were also receiving prandial RHI resulted in similar glycemic control to that provided by four daily injections of NPH insulin plus prandial RHI (same total number of insulin units) [58]. Subsequently, large, multicenter clinical trials in patients with T1DM and T2DM generally demonstrated that insulin glargine provided lower fasting blood glucose levels and less frequent hypoglycemic episodes than NPH insulin when administered alone or with premeal RHI [3, 59-63].

Given that a reduced frequency of hypoglycemia was generally observed in these studies, the target fasting blood glucose level can be lower than the target that has traditionally been used with NPH insulin [62, 64]. Patients treated with insulin glargine had less pharmacodynamic variability than patients treated with NPH insulin [65], possibly because, unlike NPH insulin [66], insulin glargine does not need to be resuspended prior to use.

Phase III study data raised no significant safety concerns for insulin glargine [51, 60, 63]. Retinopathy progression was noted in one study in patients with T2DM [67]. However, a review of all clinical trial data for insulin glargine concluded that the data did not support progression of retinopathy in patients with either T1DM or T2DM $[60,67]$. Subsequently, a large, randomized trial in patients with T2DM demonstrated that, despite slightly more severe diabetic retinopathy at baseline in the group treated with insulin glargine, the progression of retinopathy was similar in insulin glarginetreated patients and NPH-treated patients over a 5 -year period [68].
More recently, epidemiological studies suggested that insulin glargine might be associated with an increased risk of cancer $[69,70]$, but this was not confirmed by the ORIGIN trial, which showed that treatment with basal insulin glargine for more than 6 years had a neutral effect on cancers [71]. Exposure to insulin glargine is very limited as it is rapidly metabolized, primarily to the metabolite M1 [72], which mediates most of the glucodynamic effects, has slightly lower receptor binding affinity than human insulin, and does not exceed the mitogenic potential of human insulin [73].

Insulin glargine $100 \mathrm{U} / \mathrm{mL}$ is regarded as a standard-of-care basal insulin [74]. Recently, Lilly insulin glargine $\left(\right.$ Abasaglar $\left.{ }^{\circledR}\right)$, the first biosimilar insulin to receive marketing authorization in the European Union, has become available [75]. It has an identical primary amino-acid sequence to that of the insulin glargine reference product (Lantus ${ }^{\circledR}$ insulin glargine), and phase I and phase III studies (in particular ELEMENT-1 and ELEMENT-2) have demonstrated that Lilly insulin glargine has similar PK/PD profiles, efficacy, and safety to the insulin glargine reference product [76-80].

\section{BASAL-BOLUS THERAPY WITH INSULIN LISPRO AND INSULIN GLARGINE IN ADULTS WITH DIABETES}

In patients with severe insulin deficiency, insulin therapy should replace both basal and prandial insulin requirements, matching the physiologic pattern of insulin secretion as closely as possible [81]. The basal-bolus approach involves multiple daily injections (MDI), with a long-acting insulin used as the basal insulin and a rapid-acting insulin adminstered at mealtimes $[81,82]$. Long-acting insulin analogues generally reduce $\mathrm{HbA1c}$ to a similar extent to synthetic human insulins, but may be associated with less nocturnal hypoglycemia [83-85]. RAIAs are often preferred over RHI for mealtime insulin administration because they are absorbed more rapidly, can be given nearer to the meal, their action better simulates the 
physiological insulin response to meals, and they are associated with less hypoglycemia $[83,86,87]$.

Insulin glargine $100 \mathrm{U} / \mathrm{mL}$ and insulin lispro have both been available for many years, have been widely studied, and can be considered first-line options for use as the basal and bolus components, respectively, of BBT [87]. A search of the PubMed database up to October 3, 2017 was performed to identify papers about BBT involving insulin glargine $100 \mathrm{U} / \mathrm{mL}$ plus insulin lispro in patients with T1DM or T2DM that were published in English. The search was limited to human data, and the following search string was used: ((basal insulin) OR (basal bolus therapy) OR basal-plus OR basal-bolus OR basal bolus premixed) AND (insulin glargine OR Lantus OR "Lantus SoloStar" OR Basaglar OR Abasaglar OR insulin lispro OR Humalog OR basal $g$ OR flexpen OR Novorapid OR LY2963016 OR (LY2963016 AND lispro)) OR (insulin glargine biosimilar) NOT Letter. Additional papers were detected from bibliographies of the identified articles. Clinical trials, observational studies, and review articles were considered; trials could be of any duration and could involve adult patients of any age or pediatric populations. Only studies in which one treatment arm clearly comprised BBT with insulin glargine $100 \mathrm{U} / \mathrm{mL}$ plus insulin lispro (and no other insulin preparation) were retained. Comparisons with non-insulin therapy were excluded. The initial search identified 994 papers, of which 39 met the criteria for inclusion. The remainder of this section focuses on studies in adults; pediatric data are discussed later.

\section{Type 1 Diabetes Mellitus}

Insulin is the cornerstone of treatment for patients with T1DM, with the initial dosage generally based on body weight (0.4-1.0 $\mathrm{U} / \mathrm{kg} /$ day total insulin) [88]. Intensive insulin therapy ( $\geq 3$ injections per day or CSII) improves glycemic control and produces better long-term outcomes than 1-2 insulin injections per day [89-91]. Consequently, American Diabetes Association (ADA) guidelines recommend that most people with T1DM should receive either MDI ( $\geq 3$ prandial insulin injections and 1 or 2 basal insulin injections per day) or CSII [92].

\section{Insulin Glargine Plus Insulin Lispro Versus Human Insulin}

BBT using insulin glargine $100 \mathrm{U} / \mathrm{mL}$ plus insulin lispro was compared with BBT using other insulin combinations that utilized human insulin for the basal and/or prandial component in seven studies in adults with T1DM, all of which had randomized, open-label [93-97], or single-blind [98] designs (Table 1). Overall, these studies indicate that insulin glargine plus insulin lispro provides similar or better glycemic control to and less nocturnal hypoglycemia than regimens including human insulin. Overall, insulin glargine plus insulin lispro reduced HbA1c by $0.1-1.0 \% \quad(1-11 \mathrm{mmol} / \mathrm{mol})$ compared with baseline, whereas the change seen with recombinant or synthetic human insulin as the basal and/or prandial component ranged from an increase of $0.1 \%(1 \mathrm{mmol} / \mathrm{mol})$ to a reduction of $0.6 \%(7 \mathrm{mmol} / \mathrm{mol})$ (Table 1$)$. The mean number of episodes of nocturnal hypoglycemia per month was $0.53-2.0$ with insulin glargine plus insulin lispro, compared with 0.55-3.6 for regimens including human insulin (Table 1).

Only one of these studies compared insulin glargine plus insulin lispro with a combination of two human insulin products in patients with T1DM [93]. In this randomized crossover study $(n=56)$, insulin glargine plus insulin lispro provided better glycemic control than NPH insulin plus RHI, as indicated by a significantly lower HbA1c after 16 weeks of treatment [7.5\% vs. $8.0 \%$ (59 vs. $64 \mathrm{mmol} / \mathrm{mol}) ; p<0.001]$, together with an $8 \%$ lower 24 -h blood glucose AUC $(p=0.037)$. In addition, the rate of symptomatic nocturnal hypoglycemia was $44 \%$ lower with insulin glargine plus insulin lispro than with the comparator regimen (0.66 vs. 1.18 episodes/month; $p<0.001$ ) [93]. Moreover, recipients of insulin analogue therapy reported greater satisfaction with treatment [94].

The other studies ( $n=34-619$ patients) compared BBT with insulin glargine plus insulin lispro with BBT using either insulin glargine 
Table 1 Clinical trials comparing basal-bolus therapy with insulin glargine $100 \mathrm{U} / \mathrm{mL}$ plus insulin lispro with other basalbolus regimens in patients with type 1 diabetes mellitus

\begin{tabular}{|c|c|c|c|c|c|c|}
\hline & Study design; treatment & Treatment & $N$ & Mean change & from baseline & Nocturnal \\
\hline & $\begin{array}{l}\text { duration (prior treatment, } \\
\text { as specified) }\end{array}$ & & & $\begin{array}{l}\text { HbAlc (\%) } \\
{[\mathrm{mmol} / \mathrm{mol}]}\end{array}$ & $\begin{array}{l}\text { Fasting blood } \\
\text { glucose } \\
(\mathrm{mmol} / \mathrm{L})\end{array}$ & $\begin{array}{l}\text { hypoglycemia } \\
(\text { episodes per } \\
\text { mon }^{\text {a }} \text { ) }\end{array}$ \\
\hline Comparisons $\mathrm{v}$ & with human insulin & & & & & \\
\hline IGlar + LIS & vs. $\mathrm{NPH}+\mathrm{RHI}$ & & & & & \\
\hline Ashwell & $\mathrm{R}, \mathrm{OL}, \mathrm{C}, \mathrm{M} ; 16 \mathrm{wk}$ & IGlar + LIS & 56 & $\mathrm{NR}^{\mathrm{b}}$ & $\mathrm{NR}^{\mathrm{b}}$ & $0.66 \pm 0.02$ \\
\hline $\begin{array}{l}\text { et al. } \\
{[93]}\end{array}$ & $\begin{array}{l}\text { (No previous IGlar; insulin } \\
[\mathrm{MDI}] \text { for } \geq 1 \text { year })\end{array}$ & $\mathrm{NPH}+\mathrm{RHI}$ & 56 & $\mathrm{NR}^{\mathrm{b}}$ & $\mathrm{NR}^{\mathrm{b}}$ & $1.18 \pm 0.02$ \\
\hline & & & & $\mathrm{NR}^{\mathrm{b}}$ & $\mathrm{NR}^{\mathrm{b}}$ & $p<0.001$ \\
\hline IGlar vs. NPH & & & & & & \\
\hline Rossetti & $\mathrm{R}, \mathrm{OL} ; 3$ months & $\operatorname{IGlar}^{\mathrm{c}}+\mathrm{LIS}$ & 17 & $-0.4[-5]$ & NR & $2.0 \pm 0.19^{\mathrm{d}, \mathrm{e}}$ \\
\hline et al. [95] & $(\mathrm{NPH}+\mathrm{LIS}[\mathrm{MDI}])$ & $\mathrm{NPH}+$ LIS & 17 & $+0.1[+1]$ & NR & $3.6 \pm 0.4^{\mathrm{d}, \mathrm{e}}$ \\
\hline & & & & $p<0.04$ & $p<0.05$ & $p<0.05^{\mathrm{d}, \mathrm{e}}$ \\
\hline Porcellati & $\mathrm{R}, \mathrm{OL} ; 1$ year & IGlar + LIS & 61 & $-0.5[-6]$ & $\mathrm{NR}^{\mathrm{f}}$ & $1.2 \pm 0.2^{\mathrm{d}, \mathrm{e}}$ \\
\hline et al. [96] & $(\mathrm{NPH}+\mathrm{LIS}[\mathrm{MDI}])$ & $\mathrm{NPH}+$ LIS & 60 & $0[0]$ & $\mathrm{NR}^{\mathrm{f}}$ & $3.2 \pm 0.3^{\mathrm{d}, \mathrm{e}}$ \\
\hline & & & & $p<0.05$ & $\mathrm{NR}^{\mathrm{f}}$ & $p<0.05^{\mathrm{d}, \mathrm{e}}$ \\
\hline $\begin{array}{l}\text { Fulcher } \\
\text { et al. [99] }\end{array}$ & $\mathrm{R}, \mathrm{SB}, \mathrm{M} ; 30 \mathrm{wk}$ & IGlar + LIS & 62 & $\begin{aligned}- & 1.0 \\
& {[-11] }\end{aligned}$ & -3.46 & $0.22^{\mathrm{g}, \mathrm{h}}$ \\
\hline & (Insulin for $\geq 1$ year) & $\mathrm{NPH}+$ LIS & 63 & $-0.5[-6]$ & -2.34 & $0.37^{\mathrm{g}, \mathrm{h}}$ \\
\hline & & & & $p<0.01$ & $p<0.05$ & $p=0.02^{\mathrm{g}, \mathrm{h}}$ \\
\hline Bolli et al. & $\mathrm{R}, \mathrm{OL}, \mathrm{M} ; 24 \mathrm{wk}$ & IGlar + LIS & 85 & $-0.6[-7]$ & -1.56 & $0.18 \pm 0.25^{\mathrm{d}, \mathrm{i}}$ \\
\hline$[97]$ & $\begin{array}{l}(\mathrm{NPH}+\mathrm{RHI} \text { or LIS } \\
[\mathrm{MDI}])\end{array}$ & $\mathrm{NPH}+$ LIS & 90 & $-0.6[-7]$ & -0.54 & $0.16 \pm 0.25^{\mathrm{d}, \mathrm{i}}$ \\
\hline & & & & NS & $p=0.0064$ & $p=0.383^{\mathrm{d}, \mathrm{i}}$ \\
\hline Raskin & $\mathrm{R}, \mathrm{OL}, \mathrm{M} ; 16 \mathrm{wk}$ & IGlar + LIS & 310 & $-0.1[-1]$ & $-2.33 \pm 0.26$ & $1114^{\mathrm{j}}$ \\
\hline et al. [98] & $\begin{array}{l}\text { (NPH + LIS [MDI] } \\
\text { for } \geq 3 \text { months })\end{array}$ & $\mathrm{NPH}+$ LIS & 309 & $-0.1[-1]$ & $-0.69 \pm 0.26$ & $992^{\mathrm{j}}$ \\
\hline & & & & NS & $p=0.0001$ & $p=0.06^{j}$ \\
\hline LIS vs. RHI & & & & & & \\
\hline Brunetti & $\mathrm{R}, \mathrm{OL}, \mathrm{M}, \mathrm{NI} ; 16 \mathrm{wk}$ & IGlar + LIS & 193 & NR & NR & $0.022^{\mathrm{d}, \mathrm{g}}$ \\
\hline $\begin{array}{l}\text { et al. } \\
{[100]}\end{array}$ & $\begin{array}{l}\text { (NPH or IGlar }+ \text { prandial } \\
\text { insulin }[\mathrm{MDI}])\end{array}$ & IGlar + RHI & 202 & NR & NR & $0.021^{\mathrm{d}, \mathrm{g}}$ \\
\hline & & & & $N S^{k}$ & $N S^{k}$ & $p=0.742^{\mathrm{d}, \mathrm{g}}$ \\
\hline
\end{tabular}


Table 1 continued

\begin{tabular}{|c|c|c|c|c|c|}
\hline \multirow{2}{*}{$\begin{array}{l}\text { Study design; treatment } \\
\text { duration (prior treatment, } \\
\text { as specified) }\end{array}$} & \multirow[t]{2}{*}{ Treatment } & \multirow[t]{2}{*}{$N$} & \multicolumn{2}{|c|}{ Mean change from baseline } & \multirow{2}{*}{$\begin{array}{l}\text { Nocturnal } \\
\text { hypoglycemia } \\
(\text { episodes per } \\
\left.\text { mon }^{\text {a }}\right)\end{array}$} \\
\hline & & & $\begin{array}{l}\text { HbAlc (\%) } \\
{[\mathrm{mmol} / \mathrm{mol}]}\end{array}$ & $\begin{array}{l}\text { Fasting blood } \\
\text { glucose } \\
(\mathrm{mmol} / \mathrm{L})\end{array}$ & \\
\hline
\end{tabular}

Comparisons with other insulin analogues

LIS vs. GLU

\begin{tabular}{|c|c|c|c|c|c|c|}
\hline \multirow{3}{*}{$\begin{array}{c}\text { Dreyer } \\
\text { et al. } \\
{[101]}\end{array}$} & R, OL, M; 26 wk & IGlar + LIS & 333 & $-0.1[-1]$ & NR & $0.53 \pm 0.84$ \\
\hline & (Insulin for $>1$ year) & IGlar + GLU & 339 & $-0.1[-1]$ & NR & $0.55 \pm 0.94$ \\
\hline & & & & NS & NR & NR \\
\hline \multirow{3}{*}{$\begin{array}{l}\text { Kawamori } \\
\text { et al. } \\
{[102]}\end{array}$} & R, OL, M, NI; 28 wk & IGlar + LIS & 135 & $0.04[0.5]$ & NR & $0.01^{\mathrm{g}}$ \\
\hline & $(\mathrm{BBT}$ for $\geq 12 \mathrm{wk})$ & IGlar + GLU & 132 & $0.01[0.1]$ & NR & $0.00^{\mathrm{g}}$ \\
\hline & & & & $N S^{1}$ & NR & $p=0.6637^{\mathrm{g}}$ \\
\hline
\end{tabular}

$B B T$ basal-bolus therapy, $C$ crossover, $C I$ confidence interval, GLU insulin glulisine, HbAlc glycated hemoglobin, IGlar Lantus $^{\circledR}$ insulin glargine, $L I S$ insulin lispro, $M$ multicenter, $M D I$ multiple daily injections, mon months, $N$ number of patients, $N I$ noninferiority, $N P H$ neutral protamine Hagedorn insulin, $N R$ not reported, $N S$ not significant, $O L$ open-label, $R$ randomized, $R H I$ regular human insulin, $S B$ single-blind, wk weeks

${ }^{a}$ Mean \pm SE episodes of nocturnal hypoglycemia per patient per month during treatment period, unless indicated otherwise

b HbAlc value at 16 wk lower with IGlar + LIS vs. NPH + RHI [difference $-0.5 \%(6 \mathrm{mmol} / \mathrm{mol})$, $95 \% \mathrm{CI}-0.7$ to $-0.3 \%$ ( -8 to $-3 \mathrm{mmol} / \mathrm{mol}), p<0.001]$; fasting FBG value lower at 16 wk with IGlar + LIS vs. NPH + RHI (difference $-1.5 \mathrm{mmol} / \mathrm{L}, 95 \% \mathrm{CI}-2.6$ to $-0.5, p=0.005$ )

${ }^{c}$ Data shown are for patients given IGlar at bedtime. Another group received IGlar at dinnertime; there were no significant differences in HbAlc or hypoglycemia results between these two groups

d Mean number of episodes per month during the last month of treatment

e Hypoglycemia defined as blood glucose $<4.0 \mathrm{mmol} / \mathrm{L}$ irrespective of symptoms

f Mean daily blood glucose was lower with IGlar vs. NPH $(7.6 \pm 0.11 \mathrm{mmol} / \mathrm{L}$ vs. $8.1 \pm 0.22 \mathrm{mmol} / \mathrm{L}, p<0.05)$

g Severe nocturnal hypoglycemia

h Number of episodes per 100 patient days

i Serious nocturnal hypoglycemia (blood glucose $<2.3 \mathrm{mmol} / \mathrm{L}$ )

j Number of episodes during entire treatment period (16 wk)

$\mathrm{k}$ Paper states treatments did not differ with respect to HbAlc and FBG at study end

${ }^{1}$ Non-inferiority of IGlar + GLU versus IGlar + LIS was demonstrated based on analysis of covariance of the change in $\mathrm{HbAlc}$ and using a prespecified non-inferiority margin (upper $95 \% \mathrm{CI}$ limit) of $0.45 \%(5 \mathrm{mmol} / \mathrm{mol}$ ); the between-group difference in least-squares mean change was $0.1 \%$ [ $1 \mathrm{mmol} / \mathrm{mol} ; 95 \% \mathrm{CI}-0.1$ to $0.2 \%(-1$ to $2 \mathrm{mmol} / \mathrm{mol})]$

plus RHI or NPH insulin plus insulin lispro. Insulin glargine plus insulin lispro provided better control of fasting blood glucose [95-99] and/or HbA1c $[95,96,99]$ than NPH insulin plus insulin lispro, and similar glycemic control to insulin glargine plus RHI [100]. Half of these studies also found that BBT with insulin glargine plus insulin lispro reduced the frequency of total nocturnal hypoglycemia $[95,96]$ or severe nocturnal hypoglycemia [99] versus NPH insulin plus insulin lispro (mean episodes of nocturnal hypoglycemia per month $1.2-2.0$ vs. 3.2-3.6; see Table 1 for individual study results).

\section{Insulin Glargine Plus Insulin Lispro Versus Other Insulin Analogues}

When compared with BBT utilizing other insulin analogues in T1DM, BBT with insulin 
Table 2 Clinical trials comparing basal-bolus therapy with insulin glargine $100 \mathrm{U} / \mathrm{mL}$ plus insulin lispro to continuous subcutaneous insulin infusion using insulin lispro in patients with type 1 diabetes mellitus

\begin{tabular}{|c|c|c|c|c|c|c|}
\hline & \multirow{2}{*}{$\begin{array}{l}\text { Study design; duration } \\
\text { (prior treatment, as } \\
\text { specified) }\end{array}$} & \multirow[t]{2}{*}{ Treatment } & \multirow[t]{2}{*}{$N$} & \multicolumn{2}{|c|}{ Mean change from baseline } & \multirow{2}{*}{$\begin{array}{l}\text { Severe hypoglycemia } \\
\text { (episodes per patient } \\
\text { during treatment }{ }^{\mathrm{a}} \text { ) }\end{array}$} \\
\hline & & & & $\begin{array}{l}\text { HbAlc (\%) } \\
{[\mathrm{mmol} / \mathrm{mol}]}\end{array}$ & $\begin{array}{l}\text { Fasting blood } \\
\text { glucose } \\
(\mathrm{mmol} / \mathrm{L})\end{array}$ & \\
\hline \multirow{3}{*}{$\begin{array}{l}\text { Lepore et al. } \\
{[104]}\end{array}$} & OL; 1 year & IGlar + LIS & 24 & $-0.7[8]$ & NR & $0.21 \pm 0.40$ \\
\hline & $\begin{array}{l}(\mathrm{NPH}+\mathrm{RHI} \text { or } \mathrm{LIS} \\
\quad[\mathrm{MDI}] \text { for } \geq 1 \text { year })\end{array}$ & LIS CSII & 24 & $\begin{array}{l}-1.0 \\
\quad[-11]\end{array}$ & NR & $0.17 \pm 0.37$ \\
\hline & & & & NS & NR & NS \\
\hline \multirow{3}{*}{$\begin{array}{l}\text { Lepore et al. } \\
\text { [105] }\end{array}$} & OL; 1 year & IGlar + LIS & 16 & NR & NR & 0.18 \\
\hline & $\begin{array}{l}(\mathrm{NPH}+\mathrm{RHI} \text { or LIS } \\
\quad[\mathrm{MDI}] \text { for } \geq 1 \text { year })\end{array}$ & LIS CSII & 16 & NR & NR & 0.12 \\
\hline & & & & $N S^{b}$ & $N S^{b}$ & NS \\
\hline \multirow{3}{*}{$\begin{array}{l}\text { Bolli et al. } \\
\text { [106] }\end{array}$} & $\mathrm{R}, \mathrm{OL}, \mathrm{M} ; 24 \mathrm{wk}$ & IGlar + LIS & 26 & $-0.6[-7]$ & -2.7 & $35 \pm 35^{\mathrm{c}}$ \\
\hline & $\begin{array}{l}\text { (NPH-based MDI } \\
\text { regimen) }\end{array}$ & LIS CSII & 24 & $-0.7[-8]$ & -3.3 & $41 \pm 43^{c}$ \\
\hline & & & & NS & NS & $p=0.93^{c}$ \\
\hline \multirow{3}{*}{$\begin{array}{l}\text { Bruttomesso } \\
\text { et al. [107] }\end{array}$} & $\mathrm{R}, \mathrm{OL}, \mathrm{C}, \mathrm{M} ; 4$ mon & IGlar + LIS & 39 & $-0.1[-1]$ & NR & $0.1 \pm 0.4$ \\
\hline & $\begin{array}{l}\text { (LIS or ASP CSII } \\
\text { for } \geq 6 \mathrm{mon})\end{array}$ & LIS CSII & 39 & $-0.2[-2]$ & NR & $0.1 \pm 0.3$ \\
\hline & & & & NS & NR & $p=0.710$ \\
\hline \multirow{3}{*}{$\begin{array}{l}\text { Ruiz-de- } \\
\text { Adana } \\
\text { et al. [108] }\end{array}$} & $\mathrm{R}, \mathrm{OL} ; 6 \mathrm{mon}$ & IGlar + LIS & 23 & $\begin{array}{l}-0.3 \\
\quad[-3]^{\mathrm{d}}\end{array}$ & NR & $0.05 \pm 0.2$ \\
\hline & $\begin{array}{l}\text { (IGlar + LIS [MDI] } \\
\quad \text { for } 6 \text { mon })\end{array}$ & LIS CSII & 15 & $\begin{array}{l}-0.9 \\
\quad[-10]^{\mathrm{d}}\end{array}$ & NR & $0.29 \pm 1$ \\
\hline & & & & $\mathrm{NR}^{\mathrm{e}}$ & NR & $p=0.08$ \\
\hline
\end{tabular}

ASP insulin aspart, $C$ crossover, $C S I I$ continuous subcutaneous insulin infusion, IGlar Lantus ${ }^{\circledR}$ insulin glargine, $H b A 1 c$ glycated hemoglobin, $L I S$ insulin lispro, $M$ multicenter, $M D I$ multiple daily injections, mon months, $N$ number of patients, $N P H$ neutral protamine Hagedorn insulin, $N R$ not reported, $N S$ not significant, $O L$ open-label, $R$ randomized, $R H I$ regular human insulin, wk weeks

a Total number of episodes per patient during study treatment. Severe hypoglycemia, unless indicated otherwise

b Values for changes in HbA1c and FBG were not reported, but paper stated that there were no significant differences in the degree of improvement in HbAlc or fasting plasma glucose between the groups

c Overall incidence of hypoglycemia. There were also no significant differences for nonsevere, nocturnal, symptomatic, or asymptomatic hypoglycemia events

$\mathrm{d}$ All patients underwent a 6-month period of IGlar + LIS prior to randomization to receive CSII or continue with IGlar + LIS. Baseline values were obtained after this initial 6-month period of IGlar + LIS

e $p=0.03$ for comparison of HbAlc values at endpoint [IGlar + LIS $7.6 \%$ vs. CSII $7.0 \%$ (60 vs. $53 \mathrm{mmol} / \mathrm{mol})$ ] 
Table 3 Clinical trials comparing basal-bolus therapy with insulin glargine $100 \mathrm{U} / \mathrm{mL}$ plus insulin lispro to other insulin regimens in patients with type 2 diabetes mellitus

\begin{tabular}{|c|c|c|c|c|c|}
\hline \multirow{2}{*}{$\begin{array}{l}\text { Study design; duration } \\
\text { (prior treatment, as } \\
\text { specified) }\end{array}$} & \multirow[t]{2}{*}{ Treatment } & \multirow[t]{2}{*}{$N$} & \multicolumn{2}{|c|}{ Mean change from baseline } & \multirow{2}{*}{$\begin{array}{l}\text { Nocturnal } \\
\text { hypoglycemia } \\
\left(\text { episodes per year }{ }^{a}\right)\end{array}$} \\
\hline & & & $\begin{array}{l}\text { HbAlc (\%) } \\
{[\mathrm{mmol} / \mathrm{mol}]}\end{array}$ & $\begin{array}{l}\text { Fasting blood } \\
\text { glucose } \\
(\mathbf{m m o l} / \mathrm{L})\end{array}$ & \\
\hline
\end{tabular}

\begin{tabular}{|c|c|c|c|c|c|c|}
\hline \multicolumn{7}{|c|}{ Comparisons with premixed insulin } \\
\hline \multirow[t]{3}{*}{$\begin{array}{l}\text { Rosenstock } \\
\text { et al. [113] }\end{array}$} & R, OL, M, NI; 24 wk & IGlar + LIS & 187 & $\begin{array}{l}-2.1 \\
\quad[-23]\end{array}$ & -1.88 & $6.17 \pm 10.68$ \\
\hline & $\begin{array}{l}(\text { IGlar }+ \text { OAD } \\
\quad \text { for } \geq 90 \text { days })\end{array}$ & LM 50/50 t.i.d ${ }^{b}$ & 187 & $\begin{array}{l}-1.9 \\
\quad[-20]\end{array}$ & -0.74 & $4.78 \pm 7.15$ \\
\hline & & & & $p=0.021^{\mathrm{c}}$ & $\mathrm{NR}^{\mathrm{d}}$ & $p=0.139$ \\
\hline \multirow[t]{6}{*}{$\begin{array}{l}\text { Miser et al. } \\
\text { [114] }\end{array}$} & R, OL, M, NI; 24 wk & $\begin{array}{l}\text { IGlar + LIS } \\
(\operatorname{Arm~A})^{\mathrm{e}}\end{array}$ & 199 & $0.1[1]$ & NR & $3.0 \pm 13.6$ \\
\hline & $\begin{array}{c}(\mathrm{IGlar}+\mathrm{OAD} \text { or LM } \\
75 / 25+\mathrm{OAD})\end{array}$ & $\begin{array}{l}\text { LM 75/25 b.i.d. } \\
\qquad(\text { Arm A })^{\mathrm{e}}\end{array}$ & 200 & $0.0[0]$ & NR & $2.5 \pm 7.0$ \\
\hline & & & & $N R^{f}$ & NR & $p=0.657$ \\
\hline & & $\begin{array}{l}\text { IGlar + LIS } \\
(\text { Arm B })^{\mathrm{e}}\end{array}$ & 171 & $0.2[2.2]$ & NR & $2.4 \pm 6.1$ \\
\hline & & $\begin{array}{l}\text { LM 50/50 t.i.d. } \\
\quad(\text { Arm B })^{\mathrm{e}}\end{array}$ & 174 & $0.2[2.2]$ & NR & $2.5 \pm 8.1$ \\
\hline & & & & $N R^{f}$ & NR & $p=0.949$ \\
\hline \multirow[t]{3}{*}{$\begin{array}{l}\text { Jia et al. } \\
{[115]}\end{array}$} & R, OL, M, NI; 24 wk & IGlar + LIS & 202 & $\begin{array}{l}-1.1 \\
\quad[-12]\end{array}$ & -1.2 & $0.05 \pm 0.21^{\mathrm{h}}$ \\
\hline & $\begin{array}{l}\text { (PMI [human insulin-, } \\
\text { LIS- or ASP-based] } \\
\text { for } \geq 6 \mathrm{mon} \text { ) }\end{array}$ & $\begin{array}{l}\text { LM 50/50 } \\
\text { b.i.d + LM } \\
\text { 75/25 o.d. }\end{array}$ & 197 & $\begin{array}{l}-1.1 \\
\quad[-12]\end{array}$ & -0.8 & $0.03 \pm 0.09^{h}$ \\
\hline & & & & $N S^{g}$ & $p=0.002$ & $p=0.235$ \\
\hline
\end{tabular}


Table 3 continued

\begin{tabular}{|c|c|c|c|c|c|c|}
\hline & \multirow{2}{*}{$\begin{array}{l}\text { Study design; duration } \\
\text { (prior treatment, as } \\
\text { specified) }\end{array}$} & \multirow[t]{2}{*}{ Treatment } & \multirow[t]{2}{*}{$N$} & \multicolumn{2}{|c|}{ Mean change from baseline } & \multirow{2}{*}{$\begin{array}{l}\text { Nocturnal } \\
\text { hypoglycemia } \\
\left(\text { episodes per year }{ }^{a}\right)\end{array}$} \\
\hline & & & & $\begin{array}{l}\text { HbAlc (\%) } \\
{[\mathrm{mmol} / \mathrm{mol}]}\end{array}$ & $\begin{array}{l}\text { Fasting blood } \\
\text { glucose } \\
(\mathrm{mmol} / \mathrm{L})\end{array}$ & \\
\hline \multicolumn{7}{|c|}{ Comparison with other insulin analogues } \\
\hline \multirow[t]{3}{*}{$\begin{array}{l}\text { Koivisto } \\
\text { et al. [120] }\end{array}$} & R, OL, M, NI; 24 wk & IGlar + LIS & 191 & $\begin{array}{r}-1.2 \\
{[-13]}\end{array}$ & NR & $0.09^{\mathrm{h}}$ \\
\hline & $(\mathrm{OAD}+$ insulin $)$ & ILPS + LIS & 192 & $\begin{array}{l}-1.1 \\
{[-12]}\end{array}$ & NR & $0.13^{\mathrm{h}}$ \\
\hline & & & & $\mathrm{NR}^{\mathrm{i}}$ & NR & $p=0.2$ \\
\hline
\end{tabular}

ASP insulin aspart, b.i.d. twice daily, $C$ crossover, $C I$ confidence interval, $I G l a r$ Lantus ${ }^{\circledR}$ insulin glargine, $H b A 1 c$ glycated hemoglobin, ILPS insulin lispro protamine suspension, $L I S$ insulin lispro, $L M 50 / 5050 \%$ insulin lispro protamine suspension/50\% insulin lispro, $L M 75 / 2575 \%$ insulin lispro protamine suspension/25\% insulin lispro, $M$ multicenter, mon months, $N$ number of patients, $N I$ noninferiority, $N R$ not reported, $N S$ not significant, $O A D$ oral antihyperglycemic drugs, o.d. once daily, $O L$ open-label, $P M I$ premixed insulin, $R$ randomized, t.i.d. three times daily, wk weeks

${ }^{a}$ Mean \pm SD number of episodes per patient per year unless indicated otherwise

b Evening dose could be changed to LM 75/25 if necessary; this occurred in 55\% of patients

${ }^{c}$ Difference in HbAlc change from baseline to endpoint (BBT minus LM 50/50) - 0.2\% [ $2 \mathrm{mmol} / \mathrm{mol}$; $90 \% \mathrm{CI}-0.4$ to $-0.1 \%$ ( -4 to $1 \mathrm{mmol} / \mathrm{mol}$ )]. Protocol-specified lower limit of CI for noninferiority was $-0.3 \%$. Therefore, noninferiority of LM 50/50 was not demonstrated

${ }^{\mathrm{d}} p=0.013$ for comparison of fasting plasma glucose values at endpoint (IGlar + LIS $8.2 \mathrm{mmol} / \mathrm{L}$ vs. LM $50 / 50$ $8.8 \mathrm{mmol} / \mathrm{L}$ )

e Substudy of DURABLE study. During a 6-month initiation phase, patients received IGlar once daily or LM 75/25 twice daily. Patients who did not achieve glycemic control then entered the 6-month intensification substudy. Patients on IGlar entered intensification arm A and patients on LM 75/25 entered intensification arm B

${ }^{\mathrm{f}}$ Noninferiority of LM 75/25 and LM 50/50 versus BBT was demonstrated based on HbAlc values at endpoint, with $95 \%$ CI of -0.10 to $0.37 \%(-1$ to $4 \mathrm{mmol} / \mathrm{mol})$ and -0.25 to $0.25 \%(-3$ to $3 \mathrm{mmol} / \mathrm{mol})$, respectively; the noninferiority margin was set at $0.4 \%(5 \mathrm{mmol} / \mathrm{mol})$

g Noninferiority of LM 50/50-LM 75/25 vs. IGlar + LIS was demonstrated based on a noninferiority margin of $0.4 \%$ $(5 \mathrm{mmol} / \mathrm{mol})$; the between-group difference in least squares mean change was $0 \%$ [ $\mathrm{mmol} / \mathrm{mol} ; 95 \% \mathrm{CI}-0.1$ to $0.2 \%$ (- 1 to $2 \mathrm{mmol} / \mathrm{mol})]$

h Mean \pm SD number of episodes per patient per 30 days

${ }^{\mathrm{i}}$ Noninferiority of ILPS + LIS vs. IGLar + LIS was demonstrated based on analysis of covariance of the change in HbAlc and using a prespecified noninferiority margin (upper $95 \% \mathrm{CI}$ limit) of $0.4 \%$ ( $5 \mathrm{mmol} / \mathrm{mol})$; the between-group difference in least-squares mean change was $0.1 \%$ [ $1 \mathrm{mmol} / \mathrm{mol} ; 95 \% \mathrm{CI}-0.1$ to $0.3 \%$ ( 1 to $3 \mathrm{mmol} / \mathrm{mol})]$

glargine $100 \mathrm{U} / \mathrm{mL}$ plus insulin lispro provided similar glycemic control and rates of hypoglycemia to insulin glargine plus insulin glulisine in randomized clinical trials $(n=672$ and 267, respectively) (Table 1) [101, 102], and lower evening post-prandial glucose levels than insulin detemir plus insulin lispro in a crossover trial $(n=8)$, which could be due to insulin detemir having a shorter duration of action or a slower onset of action [103].

\section{Insulin Glargine Plus Insulin Lispro Versus Insulin Lispro CSII}

BBT with insulin glargine $100 \mathrm{U} / \mathrm{mL}$ plus insulin lispro was compared with CSII using insulin lispro in five open-label studies in patients with T1DM ( $n=32-50)$ (Table 2) [104-108]. The two approaches provided similar glycemic control and frequency of severe hypoglycemia in most studies [104-106], although two reported better glycemic control with CSII [107, 108]. 


\section{Type 2 Diabetes Mellitus}

Pharmacological treatment for patients with T2DM usually starts with a single oral antidiabetic agent (OAD), generally metformin [88]. If maximally titrated OAD monotherapy is inadequate, a second oral agent, a glucagon-like peptide-1 (GLP1) receptor agonist or insulin, is added [88]. The progressive decline in $\beta$-cell function that occurs in T2DM means that most patients eventually need exogenous insulin therapy in combination with other therapies $[87,88]$.

\section{Treatment Intensification Using Insulin}

Insulin is usually added to ongoing treatment with metformin and/or other OADs and possibly GLP-1 receptor agonist therapy. Rapid-acting mealtime insulin may be used as the initial insulin therapy in patients with T2DM $[109,110]$. However, it is generally more common to start with a single daily injection of basal insulin [81, 82, 88, 111]. If basal insulin alone does not provide adequate glycemic control, prandial insulin can be added, either as a full basal-bolus regimen (basal insulin with bolus insulin administered at all meals) or in a stepwise fashion, starting with the largest meal ('basal-plus' therapy) and then other meals, as necessary, to reach full BBT [11, 82, 111]. Alternatively, a GLP-1 receptor agonist might be added to basal insulin therapy as the next step $[81,88,111,112]$, or the patient could be switched from basal insulin to premixed insulin (initially administered twice daily, progressing to three times daily if necessary) $[81,88]$. The intensification option selected will depend on each patient's clinical circumstances and preferences [81].

\section{Insulin Glargine Plus Insulin Lispro Versus Premixed Insulin}

Seven studies, all using a randomized, controlled, noninferiority design, compared insulin glargine $100 \mathrm{U} / \mathrm{mL}$ plus insulin lispro with premixed insulin with the aim of establishing whether premixed insulin was noninferior to BBT. Overall, study results did not suggest any clinically relevant advantage of premixed insulin over insulin glargine plus insulin lispro [113-119].
Among three studies evaluating full BBT ( $n=374,744,402)$ (Table 3), two concluded that premixed insulin (insulin lispro mix 25/75 or $50 / 50$ ) was noninferior to BBT with insulin glargine plus insulin lispro with respect to HbA1c levels in patients who had failed to achieve glycemic control on their initial insulin regimen (in combination with OADs) $[114,115]$. The third study was unable to demonstrate noninferiority for insulin lispro $\operatorname{mix} 50 / 50$ or $75 / 25$, based on a difference in HbA1c change (BBT minus premixed) of $-0.2 \%$ [ $2 \mathrm{mmol} / \mathrm{mol} ; 90 \% \mathrm{CI}-0.4$ to $-0.1 \%$ ( -5 to $-1 \mathrm{mmol} / \mathrm{mol}$ )] after 24 weeks, against a noninferiority margin of $-0.3 \% \quad(3 \mathrm{mmol} / \mathrm{mol})$ [113]. In two of these studies, the total daily insulin dose at study end was similar with both approaches [114, 115]; in the third study, mean total insulin dose was higher in the BBT group than in the premixed-insulin group at study end (146 vs. 123 units, $p=0.002$ ) [113]. Rates of overall and nocturnal hypoglycemia and mean weight gain were similar with both treatment approaches [113-115].

The other four studies evaluated basal-plus therapy or intensification strategies involving one to three doses of insulin lispro $(n=476$, $426,344,484)$ [116-119]. In these studies, the addition of an increasing number of prandial insulin injections was effective and safe [116-119]. Three studies found premixed insulin was noninferior to basal-plus therapy with insulin glargine plus insulin lispro $[116,118,119]$. However, one study did not demonstrate noninferiority for insulin lispro mix 50/50, based on a change in HbA1c of $-1.76 \% \quad(-19 \mathrm{mmol} / \mathrm{mol}) \quad$ versus $-1.93 \%$ $(-21 \mathrm{mmol} / \mathrm{mol})$ with insulin glargine plus insulin lispro [between-group difference 0.17\% ( $2 \mathrm{mmol} / \mathrm{mol}$ ) for premixed minus basal-plus, $95 \%$ CI -0.03 to 0.37 ( -0 to $4 \mathrm{mmol} / \mathrm{mol}$ ); noninferiority margin $0.3 \% \quad(3 \mathrm{mmol} / \mathrm{mol})]$ [117]. In this study, HbA1c values were significantly lower in the basal-plus group compared with the premixed insulin group at weeks 12 and 24 weeks, although not at week 36 (study end) [117]. Total daily insulin doses, number of injections, rates of hypoglycemia, and weight changes at study end were similar with both approaches in most studies [116-119], with the 
exceptions that the total daily insulin dose and mean daily number of injections were greater [117], nocturnal hypoglycemia was more common [118], and weight gain was greater [116] with premixed than BBT in one study each.

\section{Glargine + Lispro Versus Other Insulin Analogues}

A randomized clinical trial $(n=383)$ showed that similar glycemic control was achieved with insulin glargine $100 \mathrm{U} / \mathrm{ml}$ plus insulin lispro BBT and insulin lispro protamine suspension plus insulin lispro BBT in patients with T2DM who no longer achieved glycemic targets on insulin plus OAD treatment (Table 3) [120]. More than 82\% of patients in each group received three insulin lispro injections per day throughout the study [120]. In a small crossover study involving 12 patients, BBT with insulin glargine plus insulin lispro was associated with lower pre- and postdinner glucose levels than BBT with insulin detemir plus insulin lispro [103].

\section{Insulin Administration and Titration Protocols}

Various algorithms for starting and intensifying insulin therapy in patients with T2DM are available, such as those provided by the ADA [88] and the American Association of Clinical Endocrinologists/American College of Endocrinology (AACE/ACE) [111].

Only one of the studies evaluating full BBT with insulin glargine $100 \mathrm{U} / \mathrm{mL}$ plus insulin lispro in patients with T2DM outlined the dosing algorithms used: insulin doses were adjusted weekly based on mean preprandial blood glucose level and, if a more aggressive approach was needed, total daily insulin requirement [113].

Studies that evaluated progression of insulin therapy, but not necessarily full BBT, used various algorithms based on fasting and/or preprandial blood glucose levels to adjust the dosages of insulin glargine and insulin lispro [117-119]. Importantly, patients can be trained to self-titrate bolus insulin doses safely [121]. In the 24-week AUTONOMY study, patients on optimized basal insulin glargine who were starting to add insulin lispro therapy were able to self-titrate their bolus insulin lispro safely using either of two simple algorithms, with insulin lispro adjusted every other day based on the preprandial reading from the previous 1-3 days [121].

\section{BASAL-BOLUS THERAPY WITH INSULIN LISPRO PLUS INSULIN GLARGINE IN SPECIAL POPULATIONS}

\section{Children and Adolescents}

The use of BBT with insulin glargine $100 \mathrm{U} / \mathrm{mL}$ plus insulin lispro in pediatric patients has been evaluated in a few, mostly small, trials, including two randomized open-label studies [122-124] and one noncomparative study [125]. Overall, these studies found that this regimen was effective and safe in children or adolescents with T1DM.

In a crossover study involving adolescents (aged 12-20 years, currently in puberty) with T1DM $(n=25)$ who were already receiving multiple injection regimens (not specified further), BBT with insulin glargine plus insulin lispro was at least as effective as NPH insulin plus RHI at maintaining glycemic control, and was associated with a lower incidence of nocturnal hypoglycemia [122]. In the other randomized study ( $n=175)$, insulin glargine plus insulin lispro was at least as effective as intermediate-acting NPH or lente insulin plus insulin lispro, and reduced glucose variability, in children and adolescents (aged 9-17 years) who had previously been receiving intermediateacting NPH or lente insulin [123, 124]. There was no difference in the rates of hypoglycemia [blood glucose $<2.00 \mathrm{mmol} / \mathrm{L}(<36 \mathrm{mg} / \mathrm{dL})$ or severe hypoglycemia requiring assistance] between the groups in the overall analysis [123], but in an analysis of a subset who used continuous glucose monitoring $(n=90)$, insulin glargine plus insulin lispro reduced the amount of time spent with blood glucose levels $<2.22 \mathrm{mmol} / \mathrm{L} \quad(<40 \mathrm{mg} / \mathrm{dL})$ based on such monitoring [124]. Finally, a small $(n=35)$ noncomparative study suggested that flexible 
MDI using insulin glargine plus insulin lispro (with the lispro dose adjusted according to 2-hour postprandial blood glucose measurements) improved glycemic control in preschool children who had previously been receiving twice-daily ultralente insulin plus insulin lispro [125].

\section{Elderly Patients}

No studies specifically evaluating BBT with insulin glargine $100 \mathrm{U} / \mathrm{mL}$ plus insulin lispro in elderly patients (aged $\geq 65$ years) were identified, but two studies reported subgroup analyses in patients in this age group with T2DM $[120,121]$. According to a post hoc subgroup analysis, BBT with either insulin lispro protamine suspension plus insulin lispro or insulin glargine plus insulin lispro was effective and safe in patients aged $>65$ years $(n=89)$, with mean reductions from baseline at 24 weeks in $\mathrm{HbA} 1 \mathrm{c}$ of $-1.0 \%(11 \mathrm{mmol} / \mathrm{mol})$ and $-1.2 \%$ $(13 \mathrm{mmol} / \mathrm{mol})$, respectively; these reductions were similar to those achieved in the overall population $[-1.1 \%(11 \mathrm{mmol} / \mathrm{mol})$ and $-1.2 \%$ (13 mmol/mol), respectively] [120]. The proportion of elderly patients reporting at least one hypoglycemic event was similar to that of the overall population for both treatment groups [120]. Subgroup analysis of the AUTONOMY study $(n=255 / 1112)$ showed that patients aged $\geq 65$ years on basal insulin glargine were able to self-titrate insulin lispro doses safely to achieve insulin intensification [121]. The percentages of patients aged $\geq 65$ years who achieved HbA1c targets of $\leq 7.0 \%$ $(53 \mathrm{mmol} / \mathrm{mol})$ were $58.5 \%$ and $58.0 \%$ when insulin lispro was self-titrated every day or every 3 days, respectively, and were similar to the percentages achieving this goal in the total population. There was no significant difference in the rate of hypoglycemia between algorithm groups among either the overall population or elderly patients [121].

\section{Patients with Comorbidities}

Studies have not been published that specifically evaluated BBT with insulin glargine plus insulin lispro in patients with renal disease or hepatic disease. The product characteristic summaries for insulin glargine and insulin lispro include the warning that insulin requirements may be lower in patients with renal or hepatic impairment due to reduced insulin metabolism, and lower in patients with hepatic impairment due to a reduced capacity for gluconeogenesis [31, 75], although in chronic hepatic impairment, insulin requirements may increase due to greater insulin resistance [31]. However, the summary of product characteristics for insulin lispro also states that renal and hepatic impairment do not affect the glucodynamic response to insulin lispro [31]. Studies showed that the PK and PD characteristics of insulin lispro were maintained in patients with T1DM and diabetic nephropathy [126] and that postprandial glucose levels and hypoglycemia rates were lower with insulin lispro than with RHI in patients with T2DM and compensated nonalcoholic liver disease [127]. Insulin glargine provided better glycemic control than NPH insulin in patients with T2DM on hemodialysis [128]. Overall, insulin glargine and insulin lispro appear to be suitable for use in patients with renal or hepatic disease.

No studies were identified that specifically evaluated BBT with insulin glargine plus insulin lispro in patients with cardiovascular disease. However, in patients with T2DM, the glycemic benefits of insulin glargine were unaltered by cardiovascular risk factors [129]; insulin glargine did not increase the risk of adverse cardiovascular outcomes in patients with preexisting cardiovascular disease or cardiovascular risk factors in the ORIGIN study [130, 131]; and RAIAs, including insulin lispro, had potentially more favorable effects on cardiovascular risk factors such as dyslipidemia and biomarkers of inflammation or atherosclerosis compared with RHI [132].

Two randomized, open-label studies ( $n=60$, 140) evaluated BBT with insulin glargine plus insulin lispro in hospitalized patients with diabetes $[133,134]$. In patients with T2DM on a general medicine ward, BBT with insulin glargine plus insulin lispro permitted better adherence to target insulin timing with respect to meals and reduced the percentage of patient 
days in which hypoglycemia $<70 \mathrm{mg} / \mathrm{dL}$ occurred when compared with NPH insulin plus RHI [134]. In patients with T2DM undergoing surgery, BBT using insulin glargine plus insulin lispro provided effective glycemic control, with no significant difference in postoperative glucose levels or in overall hypoglycemic episodes compared with insulin detemir plus insulin aspart [133].

\section{Other Patient Populations}

Most trials involving insulin glargine plus insulin lispro were conducted in Europe or the USA. However, ethnicity can affect the response of patients with T2DM to insulin therapy [135]. Studies and subgroup analyses have shown that BBT or basal-plus therapy with insulin glargine plus insulin lispro can provide effective glycemic control in South American [136] and East Asian populations $[115,137]$ with T2DM.

Finally, no studies appear to have specifically evaluated BBT with insulin glargine plus insulin lispro in pregnant women with diabetes; however, available data for the individual agents suggest that both are safe for use in pregnancy $[138,139]$.

\section{CONCLUSIONS}

With the growing availability of alternative insulins for use in BBT, it is timely that this article reviews the current evidence regarding BBT combining insulin glargine $100 \mathrm{U} / \mathrm{mL}$ with insulin lispro in patients with T1DM and T2DM, including its use in special populations (children, elderly, pregnant women, patients with comorbidities, and people of different ethnicities).

Insulin glargine $100 \mathrm{U} / \mathrm{mL}$ and insulin lispro have both been available for many years, have been studied extensively, and are widely used as the basal and bolus components, respectively, of BBT. Insulin lispro was the first rapid-acting insulin analogue to become available and has been evaluated in a wide range of patients, and insulin glargine is regarded as a standard-of-care basal insulin. Given the length of their availability, a substantial evidence base exists to support the efficacy and safety of these agents as BBT or basal-plus therapy in patients with T1DM and T2DM.

Clinical studies indicate that in patients with T1DM, BBT with insulin glargine plus insulin lispro provides similar or better glycemic control, and less nocturnal hypoglycemia, than BBT involving human insulin as the basal and/or prandial component. Moreover, in patients with T1DM, BBT with insulin glargine 100 $\mathrm{U} / \mathrm{mL}$ plus insulin lispro generally provides a similar level of glycemic control to that achieved with insulin lispro CSII, with similar rates of severe hypoglycemia.

In patients with T2DM receiving basal insulin, intensification of insulin therapy can generally be achieved by either initiating BBT or progressing to basal-plus therapy and then full BBT with prandial cover for all meals. Progression of insulin therapy can be achieved using various algorithms based on fasting and/or preprandial blood glucose levels to adjust the dosages of insulin glargine and insulin lispro. Algorithms for starting and intensifying insulin therapy are provided by the ADA [88] and the AACE/ACE [111]. Simple algorithms for the titration of prandial insulin lispro can facilitate patient self-management of insulin therapy. Most studies evaluating BBT with insulin glargine plus insulin lispro in patients with T2DM evaluated the noninferiority of premixed insulin versus BBT. These studies found that premixed insulin does not appear to provide any advantage over this BBT with respect to glycemic control or rates of hypoglycemia.

One of the key factors to be considered with insulin therapy is the need to achieve a balance between maintaining good glycemic control and minimizing the risk of hypoglycemic episodes. Studies such as AUTONOMY [121] demonstrate that, if well titrated, insulin therapy enables glycemic targets to be reached safely and simply.

Pooled analyses of studies involving insulin glargine or insulin lispro showed that these agents provided similar levels of efficacy and safety in elderly and young patients. In addition, insulin glargine plus insulin lispro is safe and effective in people of different ethnicities, and these insulins appear to be suitable for use 
in other special populations such as pregnant women and patients with comorbidities.

In conclusion, BBT remains a relevant option for patients with T1DM and those with T2DM requiring insulin treatment. In particular, the widely used combination of insulin glargine $100 \mathrm{U} / \mathrm{mL}$ plus insulin lispro has established efficacy and safety, and should be considered a first-line option in patients for whom BBT regimens are being considered.

\section{ACKNOWLEDGEMENTS}

Funding. Sponsorship for this study and article processing charges were funded by Eli Lilly and Company. All authors had full access to all of the data in this study and take complete responsibility for the integrity of the data and accuracy of the data analysis.

Medical Writing. The authors would like to acknowledge Katherine Croom and Caroline Spencer (Rx Communications, Mold, UK) for their medical writing assistance during the preparation of this article, funded by Eli Lilly and Company.

Authorship. All named authors meet the International Committee of Medical Journal Editors (ICMJE) criteria for authorship for this manuscript, had full access to all of the data in this study, take complete responsibility for the integrity of the data and accuracy of the data analysis, and have given final approval to the version to be published.

Disclosures. Riccardo Candido is a member of advisory boards for NovoNordisk, Sanofi Aventis, Movi, and Takeda. Kathleen Wyne is a member of advisory boards for NovoNordisk and Sanofi. Ester Romoli is a full-time employee and shareholder of Eli Lilly and Company.

Compliance with Ethics Guidelines. This article is based on previously conducted studies and does not contain any studies with human participants or animals performed by any of the authors.
Data Availability. Data sharing is not applicable to this article, as no datasets were generated or analyzed during the current study.

Open Access. This article is distributed under the terms of the Creative Commons Attribution-NonCommercial 4.0 International License (http://creativecommons.org/licenses/ by-nc/4.0/), which permits any noncommercial use, distribution, and reproduction in any medium, provided you give appropriate credit to the original author(s) and the source, provide a link to the Creative Commons license, and indicate if changes were made.

\section{REFERENCES}

1. Banting FG, Best CH, Collip JB, Campbell WR, Fletcher AA. Pancreatic extracts in the treatment of diabetes mellitus. Can Med Assoc J. 1922;12:141-6.

2. Abel JJ. Crystalline insulin. Proc Natl Acad Sci USA. 1926;12:132-6.

3. Mane K, Chaluvaraju K, Niranjan M, Zaranappa T, Manjuthej T. Review of insulin and its analogues in diabetes mellitus. J Basic Clin Pharm. 2012;3:283-93.

4. Keen H, Glynne A, Pickup JC, Viberti GC, Bilous RW, Jarrett RJ, et al. Human insulin produced by recombinant DNA technology: safety and hypoglycaemic potency in healthy men. Lancet. 1980;2:398-401.

5. Borgono CA, Zinman B. Insulins: past, present, and future. Endocrinol Metab Clin N Am. 2012;41:1-24.

6. Home PD. The pharmacokinetics and pharmacodynamics of rapid-acting insulin analogues and their clinical consequences. Diabetes Obes Metab. 2012;14:780-8.

7. Berger M. Towards more physiological insulin therapy in the 1990s. A comment. Diabetes Res Clin Pract. 1989;6:S25-31.

8. Hirsch IB. Insulin analogues. $N$ Engl $\mathrm{J}$ Med. 2005;352:174-83.

9. Vajo Z, Duckworth WC. Genetically engineered insulin analogs: diabetes in the new millenium. Pharmacol Rev. 2000;52:1-10. 
10. Tibaldi JM. Evolution of insulin: from human to analog. Am J Med. 2014;127:S25-38.

11. Ampudia-Blasco FJ, Rossetti P, Ascaso JF. Basal plus basal-bolus approach in type 2 diabetes. Diabetes Technol Ther. 2011;13(Suppl 1):S75-83.

12. Wallia A, Molitch ME. Insulin therapy for type 2 diabetes mellitus. JAMA. 2014;311:2315-25.

13. Holleman F, Hoekstra JB. Insulin lispro. N Engl J Med. 1997;337:176-83.

14. Baker EN, Blundell TL, Cutfield JF, Cutfield SM, Dodson EJ, Dodson GG, et al. The structure of $2 \mathrm{Zn}$ pig insulin crystals at $1.5 \AA$ resolution. Philos Trans R Soc Lond B Biol Sci. 1988;319:369-456.

15. Brems DN, Alter LA, Beckage MJ, Chance RE, DiMarchi RD, Green LK, et al. Altering the association properties of insulin by amino acid replacement. Protein Eng. 1992;5:527-33.

16. Slieker LJ, Brooke GS, DiMarchi RD, Flora DB, Green LK, Hoffmann JA, et al. Modifications in the B10 and B26-30 regions of the B chain of human insulin alter affinity for the human IGF-I receptor more than for the insulin receptor. Diabetologia. 1997;40(Suppl 2):S54-61.

17. Torlone E, Fanelli C, Rambotti AM, Kassi G, Modarelli F, Di Vincenzo A, et al. Pharmacokinetics, pharmacodynamics and glucose counterregulation following subcutaneous injection of the monomeric insulin analogue [Lys(B28), Pro(B29)] in IDDM. Diabetologia. 1994;37:713-20.

18. Noble SL, Johnston E, Walton B. Insulin lispro: a fast-acting insulin analog. Am Fam Physician. 1998;57(279-86):89-92.

19. Horvath K, Bock G, Regittnig W, Bodenlenz M, Wutte A, Plank J, et al. Insulin glulisine, insulin lispro and regular human insulin show comparable end-organ metabolic effects: an exploratory study. Diabetes Obes Metab. 2008;10:484-91.

20. Howey DC, Bowsher RR, Brunelle RL, Woodworth JR. [Lys(B28), Pro(B29)]-human insulin. A rapidly absorbed analogue of human insulin. Diabetes. 1994;43:396-402.

21. Heinemann L, Heise T, Wahl LC, Trautmann ME, Ampudia J, Starke AA, et al. Prandial glycaemia after a carbohydrate-rich meal in type I diabetic patients: using the rapid acting insulin analogue [Lys(B28), Pro(B29)] human insulin. Diabet Med. 1996;13:625-9.

22. Hedman CA, Lindström T, Arnqvist HJ. Direct comparison of insulin lispro and aspart shows small differences in plasma insulin profiles after subcutaneous injection in type 1 diabetes. Diabetes Care. 2001;24:1120-1.

23. von Mach MA, Brinkmann C, Hansen T, Weilemann LS, Beyer J. Differences in pharmacokinetics and pharmacodynamics of insulin lispro and aspart in healthy volunteers. Exp Clin Endocrinol Diabetes. 2002;110:416-9.

24. Heise T, Nosek L, Spitzer H, Heinemann L, Niemoller E, Frick AD, et al. Insulin glulisine: a faster onset of action compared with insulin lispro. Diabetes Obes Metab. 2007;9:746-53.

25. Arnolds S, Rave K, Hovelmann U, Fischer A, SertLangeron C, Heise T. Insulin glulisine has a faster onset of action compared with insulin aspart in healthy volunteers. Exp Clin Endocrinol Diabetes. 2010;118:662-4.

26. Bolli GB, Luzio S, Marzotti S, Porcellati F, Sert-Langeron $\mathrm{C}$, Charbonnel $\mathrm{B}$, et al. Comparative pharmacodynamic and pharmacokinetic characteristics of subcutaneous insulin glulisine and insulin aspart prior to a standard meal in obese subjects with type 2 diabetes. Diabetes Obes Metab. 2011;13:251-7.

27. Plank J, Wutte A, Brunner G, Siebenhofer A, Semlitsch B, Sommer R, et al. A direct comparison of insulin aspart and insulin lispro in patients with type 1 diabetes. Diabetes Care. 2002;25:2053-7.

28. van Bon AC, Bode BW, Sert-Langeron C, DeVries JH, Charpentier G. Insulin glulisine compared to insulin aspart and to insulin lispro administered by continuous subcutaneous insulin infusion in patients with type 1 diabetes: a randomized controlled trial. Diabetes Technol Ther. 2011;13:607-14.

29. Sanofi-Aventis. NovoRapid 100 units/ml in a vial, NovoRapid Penfill 100 units/ml, NovoRapid FlexPen 100 units/ml, NovoRapid FlexTouch 100 units/ $\mathrm{ml}$, NovoRapid PumpCart 100 units/ml. Summary of product characteristics. Paris: Sanofi-Aventis; 2017.

30. Novo Nordisk. Apidra 100 units/ml solution for injection combined. Summary of product characteristics. Bagsværd: Novo Nordisk; 2017.

31. Eli Lilly. Humalog 100 units/ml, solution for injection in vial, Humalog 100 units $/ \mathrm{ml}$, solution for injections in cartridge, Humalog KwikPen 100 units/ml, solution for injection. Summary of product characteristics. Indianapolis: Eli Lilly; 2006.

32. Uy J, Fogelfeld L, Guerra Y. Cumulative clinical experience with use of insulin lispro: critical appraisal, role in therapy, and patient considerations. Diabetes Metab Syndr Obes. 2012;5:1-10. 
33. Pfützner A, Kustner E, Forst T, Schulze-Schleppinghoff $\mathrm{B}$, Trautmann ME, Haslbeck $\mathrm{M}$, et al. Intensive insulin therapy with insulin lispro in patients with type 1 diabetes reduces the frequency of hypoglycemic episodes. Exp Clin Endocrinol Diabetes. 1996;104:25-30.

34. Anderson JH Jr, Brunelle RL, Koivisto VA, Pfutzner A, Trautmann ME, Vignati L, et al. Reduction of postprandial hyperglycemia and frequency of hypoglycemia in IDDM patients on insulin-analog treatment. Multicenter Insulin Lispro Study Group. Diabetes. 1997;46:265-70.

35. Anderson JH Jr, Brunelle RL, Keohane P, Koivisto VA, Trautmann ME, Vignati L, DiMarchi R. Mealtime treatment with insulin analog improves postprandial hyperglycemia and hypoglycemia in patients with non-insulin-dependent diabetes mellitus. Multicenter Insulin Lispro Study Group. Arch Intern Med. 1997;157(11):1249-55.

36. Lalli C, Ciofetta M, Del Sindaco P, Torlone E, Pampanelli $S$, Compagnucci $P$, et al. Long-term intensive treatment of type 1 diabetes with the shortacting insulin analog lispro in variable combination with NPH insulin at mealtime. Diabetes Care. 1999;22:468-77.

37. Annuzzi G, Del Prato S, Arcari R, Bellomo Damato A, Benzi L, Bruttomesso D, et al. Preprandial combination of lispro and NPH insulin improves overall blood glucose control in type 1 diabetic patients: a multicenter randomized crossover trial. Nutr Metab Cardiovasc Dis. 2001;11:168-75.

38. Ciofetta M, Lalli C, Del Sindaco P, Torlone E, Pampanelli S, Mauro L, et al. Contribution of postprandial versus interprandial blood glucose to HbA1c in type 1 diabetes on physiologic intensive therapy with lispro insulin at mealtime. Diabetes Care. 1999;22:795-800.

39. de la Peña A, Seger M, Soon D, Scott AJ, Reddy SR, Dobbins MA, Brown-Augsburger P, Linnebjerg $\mathrm{H}$. Bioequivalence and comparative pharmacodynamics of insulin lispro $200 \mathrm{U} / \mathrm{mL}$ relative to insulin lispro (Humalog ${ }^{\circledR}$ ) $100 \mathrm{U} / \mathrm{mL}$. Clin Pharmacol Drug Dev. 2016;5(1):69-75.

40. Eli Lilly. Humalog 200 units/ml KwikPen, solution for injection in prefilled pen. Summary of product characteristics. Indianapolis: Eli Lilly; 2016.

41. Rees TM, Lennartz AH, Ignaut DA. A comparison of glide force characteristics between 2 prefilled insulin lispro pens. J Diabetes Sci Technol. 2015;9(2):316-9.

42. Wang T, Conrad KA, van Brunt K, Rees TM. Attributes influencing insulin pen preference among caregivers and patients with diabetes who require greater than 20 units of mealtime insulin. J Diabetes Sci Technol. 2016;10(4):923-31.

43. Fineberg SE, Huang J, Brunelle R, Gulliya KS, Anderson JH Jr. Effect of long-term exposure to insulin lispro on the induction of antibody response in patients with type 1 or type 2 diabetes. Diabetes Care. 2003;26:89-96.

44. Asai M, Kodera T, Ishizeki K, Uebori S, Kashiwaya T, Itoh $\mathrm{H}$, et al. Insulin lispro reduces insulin antibodies in a patient with type 2 diabetes with immunological insulin resistance. Diabetes Res Clin Pract. 2003;61:89-92.

45. Castera V, Dutour-Meyer A, Koeppel M, Petitjean C, Darmon P. Systemic allergy to human insulin and its rapid and long acting analogs: successful treatment by continuous subcutaneous insulin lispro infusion. Diabetes Metab. 2005;31:391-400.

46. Kumar D. Lispro analog for treatment of generalized allergy to human insulin. Diabetes Care. 1997;20:1357-9.

47. Lahtela JT, Knip M, Paul R, Antonen J, Salmi J. Severe antibody-mediated human insulin resistance: successful treatment with the insulin analog lispro. A case report. Diabetes Care. 1997;20:71-3.

48. Sanofi-Aventis. Lantus (insulin glargine [rDNA origin] injection) solution for subcutaneous injection. Paris: Sanofi-Aventis; 2009.

49. Sanofi-Aventis. Lantus (summary of product characteristics). Paris: Sanofi-Aventis; 2015.

50. Bolli GB, Owens DR. Insulin glargine. Lancet. 2000;356:443-5.

51. Hilgenfeld R, Seipke G, Berchtold H, Owens DR. The evolution of insulin glargine and its continuing contribution to diabetes care. Drugs. 2014;74:911-27.

52. Owens DR, Coates PA, Luzio SD, Tinbergen JP, Kurzhals R. Pharmacokinetics of 125I-labeled insulin glargine (HOE 901) in healthy men: comparison with NPH insulin and the influence of different subcutaneous injection sites. Diabetes Care. 2000;23:813-9.

53. Luzio SD, Beck P, Owens DR. Comparison of the subcutaneous absorption of insulin glargine (Lantus) and NPH insulin in patients with type 2 diabetes. Horm Metab Res. 2003;35:434-8.

54. Le Roith D. Insulin glargine and receptor-mediated signalling: clinical implications in treating type 2 diabetes. Diabetes Metab Res Rev. 2007;23:593-9. 
55. Berti L, Kellerer M, Bossenmaier B, Seffer E, Seipke $\mathrm{G}$, Haring $\mathrm{HU}$. The long acting human insulin analog HOE 901: characteristics of insulin signalling in comparison to $\operatorname{Asp}(\mathrm{B} 10)$ and regular insulin. Horm Metab Res. 1998;30:123-9.

56. Bähr M, Kolter T, Seipke G, Eckel J. Growth promoting and metabolic activity of the human insulin analogue [GlyA21, ArgB31, ArgB32]insulin (HOE 901) in muscle cells. Eur J Pharmacol. 1997;320:259-65.

57. Heinemann L, Linkeschova R, Rave K, Hompesch B, Sedlak M, Heise T. Time-action profile of the longacting insulin analog insulin glargine (HOE901) in comparison with those of NPH insulin and placebo. Diabetes Care. 2000;23:644-9.

58. Talaulicar M, Willms B, Rosskamp R. HOE 901, a new insulin analogue, for substitution of basal insulin requirement in type I diabetes. Diabetes Stoffwechsel. 1996;5:3-6.

59. Pieber TR, Eugene-Jolchine I, Derobert E. Efficacy and safety of HOE 901 versus NPH insulin in patients with type 1 diabetes. The European Study Group of HOE 901 in type 1 diabetes. Diabetes Care. 2000;23:157-62.

60. Rosenstock J, Schwartz SL, Clark CM Jr, Park GD, Donley DW, Edwards MB. Basal insulin therapy in type 2 diabetes: 28-week comparison of insulin glargine (HOE 901) and NPH insulin. Diabetes Care. 2001;24:631-6.

61. Home PD, Rosskamp R, Forjanic-Klapproth J, Dressler A. A randomized multicentre trial of insulin glargine compared with NPH insulin in people with type 1 diabetes. Diabetes Metab Res Rev. 2005;21:545-53.

62. Yki-Järvinen H, Dressler A, Ziemen M. Less nocturnal hypoglycemia and better post-dinner glucose control with bedtime insulin glargine compared with bedtime NPH insulin during insulin combination therapy in type 2 diabetes. HOE 901/3002 Study Group. Diabetes Care. 2000;23:1130-6.

63. Rosenstock J, Park G, Zimmerman J. Basal insulin glargine (HOE 901) versus NPH insulin in patients with type 1 diabetes on multiple daily insulin regimens. U.S. Insulin Glargine (HOE 901) Type 1 Diabetes Investigator Group. Diabetes Care. 2000;23:1137-42.

64. Hanefeld M, Koehler C, Hoffmann C, Wilhelm K, Kamke W, Gerstein H. Effect of targeting normal fasting glucose levels with basal insulin glargine on glycaemic variability and risk of hypoglycaemia: a randomized, controlled study in patients with early type 2 diabetes. Diabet Med. 2010;27:175-80.
65. Lepore M, Pampanelli S, Fanelli C, Porcellati F, Bartocci L, Di Vincenzo A, et al. Pharmacokinetics and pharmacodynamics of subcutaneous injection of long-acting human insulin analog glargine, NPH insulin, and ultralente human insulin and continuous subcutaneous infusion of insulin lispro. Diabetes. 2000;49:2142-8.

66. Jehle PM, Micheler C, Jehle DR, Breitig D, Boehm BO. Inadequate suspension of neutral protamine Hagendorn (NPH) insulin in pens. Lancet. 1999;354:1604-7.

67. Davis MD, Beck RW, Home PD, Sandow J, Ferris FL. Early retinopathy progression in four randomized trials comparing insulin glargine and $\mathrm{NPH}$ [corrected] insulin. Exp Clin Endocrinol Diabetes. 2007; 115:240-3.

68. Rosenstock J, Fonseca V, McGill JB, Riddle M, Hallé J-P, Hramiak I, et al. Similar progression of diabetic retinopathy with insulin glargine and neutral protamine Hagedorn (NPH) insulin in patients with type 2 diabetes: a long-term, randomised, openlabel study. Diabetologia. 2009;52:1778-88.

69. Hemkens LG, Grouven U, Bender R, Günster C, Gutschmidt S, Selke GW, Sawicki PT. Risk of malignancies in patients with diabetes treated with human insulin or insulin analogues: a cohort study. Diabetologia. 2009;52(9):1732-44.

70. Jonasson JM, Ljung R, Talbäck M, Haglund B, Gudbjörnsdòttir S, Steineck G. Insulin glargine use and short-term incidence of malignancies-a population-based follow-up study in Sweden. Diabetologia. 2009;52(9):1745-54.

71. Bordeleau L, Yakubovich N, Dagenais GR, Rosenstock J, Probstfield J, Chang YuP, et al. The association of basal insulin glargine and/or n-3 fatty acids with incident cancers in patients with dysglycemia. Diabetes Care. 2014;37:1360-6.

72. Bolli GB, Hahn AD, Schmidt R, Eisenblaetter T, Dahmen R, Heise T, Becker RH. Plasma exposure to insulin glargine and its metabolites M1 and M2 after subcutaneous injection of therapeutic and supratherapeutic doses of glargine in subjects with type 1 diabetes. Diabetes Care. 2012;35(12):2626-30.

73. Sommerfeld MR, Müller G, Tschank G, Seipke G, Habermann P, Kurrle R, Tennagels N. In vitro metabolic and mitogenic signaling of insulin glargine and its metabolites. PLoS One. 2010;5(3):e9540.

74. Charbonnel PB. Basal insulin intensification in type 2 diabetes: a key role for GLP-1 receptor agonists. Diabetes Metab. 2015;41(6 Suppl 1):6S1-2. 
75. Eli Lilly. ABASAGLAR 100 units/mL solution: summary of product characteristics. Indianapolis: Eli Lilly; 2014.

76. Linnebjerg H, Lam ECQ, Seger ME, Coutant D, Chua L, Chong CL, et al. Comparison of the pharmacokinetics and pharmacodynamics of LY2963016 insulin glargine and EU- and US-approved versions of lantus insulin glargine in healthy subjects: three randomized euglycemic clamp studies. Diabetes Care. 2015;38:2226-33.

77. Linnebjerg H, Lam EC, Zhang X, Seger ME, Coutant D, Chua L, et al. Duration of action of two insulin glargine products, LY2963016 insulin glargine and lantus insulin glargine, in subjects with type 1 diabetes mellitus. Diabetes Obes Metab. 2017;19:33-9.

78. Ilag LL, Deeg MA, Costigan T, Hollander P, Blevins TC, Edelman SV, et al. Evaluation of immunogenicity of LY2963016 insulin glargine compared with Lantus ${ }^{\circledR}$ insulin glargine in patients with type 1 or type 2 diabetes mellitus. Diabetes Obes Metab. 2016;18:159-68.

79. Blevins TC, Dahl D, Rosenstock J, Ilag LL, Huster WJ, Zielonka JS, et al. Efficacy and safety of LY2963016 insulin glargine compared with insulin glargine $\left(\right.$ Lantus $^{\circledR}$ ) in patients with type 1 diabetes in a randomized controlled trial: the ELEMENT 1 study. Diabetes Obes Metab. 2015;17:726-33.

80. Rosenstock J, Hollander P, Bhargava A, Ilag LL, Pollom RK, Zielonka JS, et al. Similar efficacy and safety of LY2963016 insulin glargine and insulin glargine (Lantus ${ }^{\circledR}$ ) in patients with type 2 diabetes who were insulin-naive or previously treated with insulin glargine: a randomized, double-blind controlled trial (the ELEMENT 2 study). Diabetes Obes Metab. 2015;17:734-41.

81. Pettus J, Santos Cavaiola T, Tamborlane WV, Edelman S. The past, present, and future of basal insulins. Diabetes Metab Res Rev. 2016;32:478-96.

82. Meneghini LF. Intensifying insulin therapy: what options are available to patients with type 2 diabetes? Am J Med. 2013;126:S28-37.

83. Waugh N, Cummins E, Royle P, Clar C, Marien M, Richter B, et al. Newer agents for blood glucose control in type 2 diabetes: systematic review and economic evaluation. Health Technol Assess. 2010;14:1-248.

84. DeWitt DE, Hirsch IB. Outpatient insulin therapy in type 1 and type 2 diabetes mellitus: scientific review. JAMA. 2003;289:2254-64.

85. Rosenstock J, Dailey G, Massi-Benedetti M, Fritsche A, Lin Z, Salzman A. Reduced hypoglycemia risk with insulin glargine: a meta-analysis comparing insulin glargine with human NPH insulin in type 2 diabetes. Diabetes Care. 2005;28:950-5.

86. Garg S, Ampudia-Blasco FJ, Pfohl M. Rapid-acting insulin analogues in basal-bolus regimens in type 1 diabetes mellitus. Endocr Pract. 2010;16:486-505.

87. Horton ES. Defining the role of basal and prandial insulin for optimal glycemic control. J Am Coll Cardiol. 2009;53:S21-7.

88. American Diabetes Association. 8. Pharmacologic approaches to glycemic treatment. Diabetes Care. 2017;40(Suppl 1):S64-74.

89. Orchard TJ, Nathan DM, Zinman B, Writing Group for the DCCT/EDIC Research Group, et al. Association between 7 years of intensive treatment of type 1 diabetes and long-term mortality. JAMA. 2015;313:45-53.

90. Diabetes Control and Complications Trial Research Group, Nathan DM, Genuth S, Lachin J, Cleary P, et al. The effect of intensive treatment of diabetes on the development and progression of long-term complications in insulin-dependent diabetes mellitus. N Engl J Med. 1993;329:977-86.

91. Nathan DM, Cleary PA, Backlund JY, Genuth SM, Lachin JM, Orchard TJ, et al. Intensive diabetes treatment and cardiovascular disease in patients with type 1 diabetes. $\mathrm{N}$ Engl $\mathrm{J}$ Med. 2005;353:2643-53.

92. Chiang JL, Kirkman MS, Laffel LM, Peters AL. Type 1 diabetes through the life span: a position statement of the American Diabetes Association. Diabetes Care. 2014;37:2034-54.

93. Ashwell SG, Amiel SA, Bilous RW, Dashora U, Heller SR, Hepburn DA, et al. Improved glycaemic control with insulin glargine plus insulin lispro: a multicentre, randomized, cross-over trial in people with type 1 diabetes. Diabet Med. 2006;23:285-92.

94. Ashwell SG, Bradley C, Stephens JW, Witthaus E, Home PD. Treatment satisfaction and quality of life with insulin glargine plus insulin lispro compared with NPH insulin plus unmodified human insulin in individuals with type 1 diabetes. Diabetes Care. 2008;31:1112-7.

95. Rossetti P, Pampanelli S, Fanelli C, Porcellati F, Costa E, Torlone E, et al. Intensive replacement of basal insulin in patients with type 1 diabetes given rapid-acting insulin analog at mealtime: a 3-month comparison between administration of NPH insulin four times daily and glargine insulin at dinner or bedtime. Diabetes Care. 2003;26:1490-6.

96. Porcellati F, Rossetti P, Pampanelli S, Fanelli CG, Torlone E, Scionti L, et al. Better long-term 
glycaemic control with the basal insulin glargine as compared with NPH in patients with type 1 diabetes mellitus given meal-time lispro insulin. Diabet Med. 2004;21:1213-20.

97. Bolli GB, Songini M, Trovati M, Del Prato S, Ghirlanda $G$, Cordera $R$, et al. Lower fasting blood glucose, glucose variability and nocturnal hypoglycaemia with glargine vs NPH basal insulin in subjects with type 1 diabetes. Nutr Metab Cardiovasc Dis. 2009; 19:571-9.

98. Raskin P, Klaff L, Bergenstal R, Halle JP, Donley D, Mecca T. A 16-week comparison of the novel insulin analog insulin glargine (HOE 901) and NPH human insulin used with insulin lispro in patients with type 1 diabetes. Diabetes Care. 2000;23:1666-71.

99. Fulcher GR, Gilbert RE, Yue DK. Glargine is superior to neutral protamine Hagedorn for improving glycated haemoglobin and fasting blood glucose levels during intensive insulin therapy. Intern Med J. 2005;35:536-42.

100. Brunetti P, Muggeo M, Cattin L, Arcangeli A, Pozzilli $\mathrm{P}$, Provenzano $\mathrm{V}$, et al. Incidence of severe nocturnal hypoglycemia in patients with type 1 diabetes treated with insulin lispro or regular human insulin in addition to basal insulin glargine. Nutr Metab Cardiovasc Dis. 2010;20:519-26.

101. Dreyer M, Prager R, Robinson A, Busch K, Ellis G, Souhami E, et al. Efficacy and safety of insulin glulisine in patients with type 1 diabetes. Horm Metab Res. 2005;37:702-7.

102. Kawamori R, Kadowaki T, Ishii H, Iwasaki M, Iwamoto Y. Efficacy and safety of insulin glulisine in Japanese patients with type 1 diabetes mellitus. Diabetes Obes Metab. 2009;11:891-9.

103. Kato T, Tokubuchi I, Muraishi K, Sato S, Kato T, Hara K, et al. Distinct pharmacodynamics of insulin glargine and insulin detemir: crossover comparison in type 1 and type 2 diabetic patients on basal-bolus regimen. Diabetes Res Clin Pract. 2010;90:e64-6.

104. Lepore G, Dodesini AR, Nosari I, Trevisan R. Effect of continuous subcutaneous insulin infusion vs multiple daily insulin injection with glargine as basal insulin: an open parallel long-term study. Diabetes Nutr Metab. 2004;17:84-9.

105. Lepore G, Dodesini AR, Nosari I, Trevisan R. Both continuous subcutaneous insulin infusion and a multiple daily insulin injection regimen with glargine as basal insulin are equally better than traditional multiple daily insulin injection treatment. Diabetes Care. 2003;26:1321-2.

106. Bolli GB, Kerr D, Thomas R, Torlone E, Sola-Gazagnes $\mathrm{A}$, Vitacolonna $\mathrm{E}$, et al. Comparison of a multiple daily insulin injection regimen (basal once-daily glargine plus mealtime lispro) and continuous subcutaneous insulin infusion (lispro) in type 1 diabetes: a randomized open parallel multicenter study. Diabetes Care. 2009;32:1170-6.

107. Bruttomesso D, Crazzolara D, Maran A, Costa S, Dal Pos M, Girelli A, et al. In type 1 diabetic patients with good glycaemic control, blood glucose variability is lower during continuous subcutaneous insulin infusion than during multiple daily injections with insulin glargine. Diabet Med. 2008;25:326-32.

108. Ruiz-de-Adana MS, Dominguez-Lopez ME, Gonzalez-Molero I, Machado A, Martin V, Cardona I, et al. Comparison between a multiple daily insulin injection regimen (basal once-daily glargine plus mealtime lispro) and continuous subcutaneous insulin infusion (lispro) using continuous glucose monitoring in metabolically optimized type 1 diabetes patients: a randomized open-labelled parallel study. Med Clin (Barc). 2016;146:239-46.

109. Yki-Järvinen $\mathrm{H}$, Kotronen A. Is there evidence to support use of premixed or prandial insulin regimens in insulin-naive or previously insulin-treated type 2 diabetic patients? Diabetes Care. 2013;36(Suppl 2):S205-11.

110. Bretzel RG, Nuber U, Landgraf W, Owens DR, Bradley C, Linn T. Once-daily basal insulin glargine versus thrice-daily prandial insulin lispro in people with type 2 diabetes on oral hypoglycaemic agents (APOLLO): an open randomised controlled trial. Lancet. 2008;371:1073-84.

111. Garber AJ, Abrahamson MJ, Barzilay JI, Blonde L, Bloomgarden ZT, Bush MA, et al. Consensus statement by the American Association of Clinical Endocrinologists and American College of Endocrinology on the comprehensive type 2 diabetes management algorithm-2016 executive summary. Endocr Pract. 2016;22:84-113.

112. Porcellati F, Lucidi P, Bolli GB, Fanelli CG. GLP-1 RAs as compared to prandial insulin after failure of basal insulin in type 2 diabetes: lessons from the $4 \mathrm{~B}$ and Get-Goal DUO 2 trials. Diabetes Metab. 2015;41:6s16-20.

113. Rosenstock J, Ahmann AJ, Colon G, Scism-Bacon J, Jiang H, Martin S. Advancing insulin therapy in type 2 diabetes previously treated with glargine plus oral agents: prandial premixed (insulin lispro protamine suspension/lispro) versus basal/bolus (glargine/lispro) therapy. Diabetes Care. 2008;31:20-5.

114. Miser WF, Arakaki R, Jiang H, Scism-Bacon J, Anderson PW, Fahrbach JL. Randomized, open-label, parallel-group evaluations of basal-bolus therapy versus insulin lispro premixed therapy in 
patients with type 2 diabetes mellitus failing to achieve control with starter insulin treatment and continuing oral antihyperglycemic drugs: a noninferiority intensification substudy of the DURABLE trial. Clin Ther. 2010;32:896-908.

115. Jia W, Xiao X, Ji Q, Ahn KJ, Chuang LM, Bao Y, et al. Comparison of thrice-daily premixed insulin (insulin lispro premix) with basal-bolus (insulin glargine once-daily plus thrice-daily prandial insulin lispro) therapy in east Asian patients with type 2 diabetes insufficiently controlled with twice-daily premixed insulin: an open-label, randomised, controlled trial. Lancet Diabetes Endocrinol. 2015;3:254-62.

116. Tinahones FJ, Gross JL, Onaca A, Cleall S, Rodriguez A. Insulin lispro low mixture twice daily versus basal insulin glargine once daily and prandial insulin lispro once daily in patients with type 2 diabetes requiring insulin intensification: a randomized phase IV trial. Diabetes Obes Metab. 2014;16:963-70.

117. Jain SM, Mao X, Escalante-Pulido M, Vorokhobina N, Lopez I, Ilag LL. Prandial-basal insulin regimens plus oral antihyperglycaemic agents to improve mealtime glycaemia: initiate and progressively advance insulin therapy in type 2 diabetes. Diabetes Obes Metab. 2010;12:967-75.

118. Giugliano D, Tracz M, Shah S, Calle-Pascual A, Mistodie C, Duarte R, et al. Initiation and gradual intensification of premixed insulin lispro therapy versus basal mealtime insulin in patients with type 2 diabetes eating light breakfasts. Diabetes Care. 2014;37:372-80.

119. Bowering K, Reed VA, Felicio JS, Landry J, Ji L, Oliveira J. A study comparing insulin lispro mix 25 with glargine plus lispro therapy in patients with type 2 diabetes who have inadequate glycaemic control on oral anti-hyperglycaemic medication: results of the PARADIGM study. Diabet Med. 2012;29:e263-72.

120. Koivisto V, Cleall S, Pontiroli AE, Giugliano D. Comparison of insulin lispro protamine suspension versus insulin glargine once daily in basal-bolus therapies with insulin lispro in type 2 diabetes patients: a prospective randomized open-label trial. Diabetes Obes Metab. 2011;13:1149-57.

121. Edelman SV, Liu R, Johnson J, Glass LC. AUTONOMY: the first randomized trial comparing two patient-driven approaches to initiate and titrate prandial insulin lispro in type 2 diabetes. Diabetes Care. 2014;37:2132-40.

122. Murphy NP, Keane SM, Ong KK, Ford-Adams M, Edge JA, Acerini CL, et al. Randomized cross-over trial of insulin glargine plus lispro or NPH insulin plus regular human insulin in adolescents with type 1 diabetes on intensive insulin regimens. Diabetes Care. 2003;26:799-804.

123. Chase HP, Arslanian S, White NH, Tamborlane WV. Insulin glargine versus intermediate-acting insulin as the basal component of multiple daily injection regimens for adolescents with type 1 diabetes mellitus. J Pediatr. 2008;153:547-53.

124. White NH, Chase HP, Arslanian S, Tamborlane WV. Comparison of glycemic variability associated with insulin glargine and intermediate-acting insulin when used as the basal component of multiple daily injections for adolescents with type 1 diabetes. Diabetes Care. 2009;32:387-93.

125. Alemzadeh R, Berhe T, Wyatt DT. Flexible insulin therapy with glargine insulin improved glycemic control and reduced severe hypoglycemia among preschool-aged children with type 1 diabetes mellitus. Pediatrics. 2005;115:1320-4.

126. Rave K, Heise T, Pfutzner A, Heinemann L, Sawicki PT. Impact of diabetic nephropathy on pharmacodynamic and pharmacokinetic properties of insulin in type 1 diabetic patients. Diabetes Care. 2001;24:886-90.

127. Gentile S, Guarino G, Strollo F, Romano M, Genovese $S$, Masarone $M$, et al. Lispro insulin in people with non-alcoholic liver cirrhosis and type 2 diabetes mellitus. Diabetes Res Clin Pract. 2016;113:179-86.

128. Toyoda M, Kimura M, Yamamoto N, Miyauchi M, Umezono T, Suzuki D. Insulin glargine improves glycemic control and quality of life in type 2 diabetic patients on hemodialysis. J Nephrol. 2012;25:989-95.

129. Blonde L, Baron MA, Zhou R, Banerji MA. Efficacy and risk of hypoglycemia with use of insulin glargine or comparators in patients with cardiovascular risk factors. Postgrad Med. 2014;126:172-89.

130. Mellbin LG, Ryden L, Riddle MC, Probstfield J, Rosenstock J, Diaz R, et al. Does hypoglycaemia increase the risk of cardiovascular events? A report from the ORIGIN trial. Eur Heart J. 2013;34:3137-44.

131. Lonn EM, Bosch J, Diaz R, Lopez-Jaramillo P, Ramachandran A, Hancu N, et al. Effect of insulin glargine and n-3FA on carotid intima-media thickness in people with dysglycemia at high risk for cardiovascular events: the Glucose Reduction and Atherosclerosis Continuing Evaluation study (ORIGIN-GRACE). Diabetes Care. 2013;36:2466-74. 
132. Maiti R, Jaida J, Leander PJ, Irfanuddin M, Ahmed I, Palani A. Cardioprotective role of insulin: advantage analogues. J Res Med Sci. 2012;17:642-8.

133. Ahmad M, Wafai ZA, Mathur SK, Lodha S, Khan ZY, Bansal A. Evaluation of glycemia control achieved by glargine and lispro versus detemir and aspart insulin regimes in type 2 diabetics undergoing surgery. Open Cardiovasc Med J. 2015;9:58-61.

134. Guerra YS, Lacuesta EA, Yrastorza R, Miernik J, Shakya N, Fogelfeld L. Insulin injections in relation to meals in the hospital medicine ward: comparison of 2 protocols. Endocr Pract. 2011;17:737-46.

135. Ji L, Min KW, Oliveira J, Lew T, Duan R. Comparison of efficacy and safety of two starting insulin regimens in non-Asian, Asian Indian, and East Asian patients with type 2 diabetes: a post hoc analysis of the PARADIGM study. Diabetes Metab Syndr Obes. 2016;9:243-9.

136. Rojas A, Sposetti G, Gross JL, Barbieri DE, Duan R, Linetzky $\mathrm{B}$, et al. Insulin lispro low mixture twice daily vs basal insulin glargine once daily and prandial insulin lispro once daily as insulin intensification strategies in patients with type 2 diabetes: Latin American subpopulation analysis of a randomized trial. Diabetol Metab Syndr. 2016;8:69.

137. Jeong IK, Chung $\mathrm{CH}$, Zhou Z, Han JH, Duan R, Edralin DM, et al. Comparison of insulin intensification strategies with insulin lispro low mixture twice daily versus basal insulin glargine and prandial insulin lispro once daily in East Asian and Caucasian patients with type 2 diabetes mellitus. J Diabetes. 2017;9:396-404.

138. de Valk HW, Visser GH. Insulin during pregnancy, labour and delivery. Best Pract Res Clin Obstet Gynaecol. 2011;25:65-76.

139. Lepercq J, Lin J, Hall GC, Wang E, Dain MP, Riddle $\mathrm{MC}$, et al. Meta-analysis of maternal and neonatal outcomes associated with the use of insulin glargine versus NPH insulin during pregnancy. Obstet Gynecol Int. 2012;2012:649070. 